\title{
Myofibroblast Senescence Promotes Arrhythmogenic Remodeling in the Aged $\underline{\text { Infarcted Rabbit Heart }}$
}

Brett C. Baggett ${ }^{1,2}$, Kevin R. Murphy ${ }^{1,2}$, Elif Sengun ${ }^{2}$, Eric $\mathrm{Mi}^{1,2}$, Yueming Cao ${ }^{1,2}$, Nilufer Turan ${ }^{2}$, Yichun Lu², Lorraine Scofield ${ }^{2}$, Tae Yun Kim² ${ }^{2}$ Anatoli Y. Kabakov ${ }^{1,2}$, Peter Bronk ${ }^{2}$, Zhilin Qu ${ }^{3}$, Patrizia Camelliti ${ }^{4}$, Patrycja Dubielecka $^{1,5}$, Dmitry Terentyev ${ }^{2}$, Federica Del Monte ${ }^{6}$, Bum-Rak Choi ${ }^{2}$, John Sedivy ${ }^{1}$, Gideon Koren ${ }^{1,2}$

Affiliation footnotes: 1) Brown University, Providence, RI, 2) Cardiovascular Research Center, Rhode Island Hospital, Providence, RI, 3) School of Medicine, UCLA, Los Angeles, CA, 4) School of Biosciences and Medicine, University of Surrey, Guildford, UK, 5) Department of Hematology, Rhode Island Hospital, 6) Medical University of South Carolina, Charleston,

Conflict of Interest Statement: The authors have declared that no conflict of interest exists 


\section{Abstract}

Progressive tissue remodeling after myocardial infarction (MI) promotes cardiac arrhythmias. This process is well studied in young animals, but little is known about pro-arrhythmic changes in aged animals. Senescent cells accumulate with age and accelerate age-associated diseases. Senescent cells interfere with cardiac function and outcome post-MI with age, but studies have not been performed in large animals, and the mechanisms are unknown. Here, we investigated the role of senescence in regulating inflammation, fibrosis, and arrhythmogenesis in young and aged infarcted rabbits. Aged rabbits exhibited increased peri-procedural mortality and arrhythmogenic electrophysiological remodeling at the infarct border zone (IBZ) compared to young rabbits. Studies of the aged infarct zone revealed persistent myofibroblast senescence and increased inflammatory signaling over a twelve-week timecourse. Senescent IBZ myofibroblasts in aged rabbits appear to be coupled to myocytes, and our computational modeling showed that senescent myofibroblastcardiomyocyte coupling prolongs action potential duration (APD) and facilitates conduction block permissive of arrhythmias. Aged infarcted human ventricles show levels of senescence consistent with aged rabbits, and senescent myofibroblasts also couple to IBZ myocytes. Our findings suggest that senolytic drugs may mitigate arrhythmias post-MI. 


\section{Introduction}

Sudden Cardiac Death (SCD) remains the leading cause of death worldwide and is responsible for up to $20 \%$ of all deaths in the US [1,2]. The incidence of SCD increases exponentially with age, which poses a growing problem as the portion of the US population 65 years or older is estimated to increase to $15 \%$ by 2030 $[1,3]$. The vast majority of SCDs are caused by ventricular tachycardia/ventricular fibrillation (VT/VF) subsequent to myocardial infarction (MI) [4]. Immediate clinical reperfusion interventions can limit the extent of acute ischemic injury and acute arrhythmic mortality post-MI, however progressive tissue remodeling in the days-to-years post-MI can establish a substrate and trigger for potentially lethal arrhythmias [5]. This tissue remodeling process is well described in young animals [6], but less is known about what changes to this process occur with age and how these changes increase risk of arrhythmia initiation and propagation.

We have previously characterized the efficacy of the New Zealand White rabbit as a model of the aging human heart. We showed that the aging rabbit heart recapitulates cardiac dysfunctions observed in aging humans, including aortic stiffening, reduced ventricular compliance, increased interstitial fibrosis, abnormal conduction in the His-Purkinje system, and lower thresholds for induced ventricular arrhythmias [7]. We also demonstrated defects in autophagy and mitochondrial function in isolated aged rabbit myocytes resulting in increased mitochondrial reactive oxygen species which oxidized the ryanodine receptor and promoted spontaneous $\mathrm{Ca}^{2+}$ release $[8,9]$. To better investigate the interaction between post-MI tissue remodeling and arrhythmogenesis with age, we have established a minimally invasive surgical procedure to reproducibly infarct the rabbit heart regardless of age or variable coronary anatomy, whereby an embolic coil occludes the left coronary artery [10]. Infarction by this method results in electrophysiological remodeling characteristic of human MI, including functional reentry anchored at the infarct border zone (IBZ), reduction in ejection fraction, and lower thresholds for monomorphic VT induced by programmed stimulation [10, 11].

Cellular senescence is a stress response characterized by irreversible proliferation arrest, resistance to apoptosis, and secretion of a collection of inflammatory cytokines, growth factors, and proteases termed the senescence-associated secretory phenotype (SASP) [12-14]. Transient senescence plays beneficial roles in 
young animals in wound healing by limiting fibrosis, in tumor suppression, and in development [15-17]. However, senescent cells accumulate with age in many tissues and accelerate many aging-associated pathologies in a paracrine manner via SASP-mediated chronic inflammatory signaling and/or in a juxtacrine manner via transfer of intracellular proteins to neighboring cells [18-23]. Genetic or pharmacological elimination of senescent cells with age slows the onset and progression of many aging pathologies, including atherosclerosis, diabetes, idiopathic pulmonary fibrosis, osteoarthritis, and neurodegenerative diseases [2432].

In the adult infarcted wild-type mouse heart, senescent cells arise in the scar and IBZ around day 3 post-MI and are largely cleared by day $7[21,33]$. Most of these senescent cells are cardiac myofibroblasts, the main cell type responsible for secreting extracellular matrix components forming the scar. This observation is consistent with epidermal wound studies in mice, suggesting a role for transient myofibroblast senescence as a normal part of the wound healing process in limiting fibrosis [17]. Infarction of adult constitutive p53 KO mice, in which senescence induction is impeded, results in increased fibrosis and decreased inflammation in the scar 7 days post-MI [33]. In a cell culture model of isolated mouse cardiac fibroblasts, senescent cardiac fibroblasts limit the proliferation of non-senescent cardiac fibroblasts in a juxtacrine but not paracrine fashion [21]. These results suggest a beneficial role for transient myofibroblast senescence in limiting fibrosis post-MI. Conversely, insights towards a pathological role of chronic cardiac senescence come from aging mouse models of MI. Treatment of aged mice with the senolytic drug navitoclax (i.e. a drug that specifically eliminates senescent cells) before or after MI significantly reduces senescence burden, improves cardiac function, mitigates cardiac remodeling, and reduces scar size post-MI, although the effects on arrhythmias has not been studied to our knowledge [34]. These results suggest that acute induction of senescence followed by timely clearance limits excess scarring, but persistence of cardiac senescence as seen with age can promote cardiac fibrogenesis and worsen cardiac function and might lead to increased risk of arrhythmias.

Potential pro-arrhythmic effects of senescent cells could occur through a paracrine fashion via SASP components. Indeed, treatment of isolated rat and mouse cardiomyocytes with exogenous SASP components including IL-6, IL-1 $\beta$, and TNF- $\alpha$ have pro-arrhythmic effects in ion channel remodeling [35, 36]. Senescent myofibroblasts might be able to interfere with cardiomyocyte electrophysiology from relatively long distances 
through chronic inflammatory signaling. Alternatively, the direct cell-cell coupling via gap junctions of senescent myofibroblasts to cardiomyocytes at the IBZ might alter their electrophysiology more than coupling with nonsenescent myofibroblasts. Such a process would establish regional heterogeneities in ion channel activity, action potential duration (APD), and other electrophysiological factors anchored at the IBZ that would permit reentrant current and therefore VT/VF.

We hypothesized that aged infarcted rabbits experience a persistence of senescent myofibroblasts in the scar and IBZ compared to young, and that these senescent myofibroblasts act in a paracrine or juxtacrine fashion to induce proarrhythmic remodeling in IBZ cardiomyocytes. Here, we demonstrate that aged rabbits compared to young exhibit increased peri-procedural deaths mostly due to VT/VF, consistent with prolongation of APD and alternans at the IBZ associated with higher frequency VF. We observed no difference in the size of the scar or IBZ geometry over the first twelve weeks post-MI between young and aged rabbits. However, whereas senescent cells in the young rabbit scar were largely cleared by 3 weeks post-MI, senescence of mostly myofibroblasts remained high in the aged rabbits up to 12 weeks post-MI and correlated with increased local expression of inflammatory cytokines. In ventricular tissue samples from aged infarcted human patients, we observed elevated levels of senescence markers, and most senescent cells appeared to be myofibroblasts. Using primary adult rabbit cardiac fibroblasts, we induced senescence via treatment with the drug etoposide. With this method, we did not observe any effect of conditioned media from senescent cardiac fibroblasts on the $\mathrm{I}_{\mathrm{Kr}}$ current or APD of treated 3-week-old rabbit myocytes compared to conditioned media from proliferating cardiac fibroblasts. However, in both aged rabbit and human cardiac tissue, we observed senescent cardiac myofibroblasts in close proximity to IBZ myocytes, with the gap junction protein $\mathrm{Cx} 43$ appearing to couple the cells. Previous studies in rabbits have demonstrated a role for Cx43 in the coupling of myocytes and fibroblasts as evidenced by dye transfer and optogenetic studies [37-39]. Based on our electrophysiological and volumetric measurements of senescent and non-senescent fibroblasts, we performed computational modeling which suggested that coupling of myocytes with senescent cardiac myofibroblasts would result in prolongation of APD and lower thresholds for conduction block. Altogether, our results indicate a pathological role for the persistence of senescent cardiac myofibroblasts in directly exacerbating arrhythmogenesis at least via a direct cell-cell interaction through gap junctions with age post-MI. These findings suggest targeted elimination of 
senescent cells post-MI could be an effective therapeutic method to mitigate senescence burden and combat arrhythmias in aged infarcted individuals.

\section{$\underline{\text { Results }}$}

\section{Aged Infarcted Rabbits Exhibit Increased Incidence of Peri-Procedural Arrhythmias}

A total of 73 young ( $\leq 1$ year) and 99 aged ( $\geq 4$ years) female New Zealand White rabbits were subjected to minimally-invasive coil embolization of the left coronary artery to induce MI of the apical left ventricular free wall (Figure 1). The average age for young rabbits was 7.8 months and for aged rabbits was 4.8 years. Aged rabbits exhibited significantly higher peri-procedural mortality compared to young rabbits $(36 \%$ of aged rabbits versus $19 \%$ of young rabbits, $\mathrm{p}=0.0120$ ), with most young and aged peri-procedural deaths attributed to VT/NF. We observed no significant difference in SCD in the acute (3-72hrs) or chronic ( $\geq 72 \mathrm{hrs})$ periods post-MI between young and aged rabbits. Aged rabbits exhibited a significantly higher incidence of peri-procedural lethal or nonlethal VT/VF ( $44 \%$ of aged rabbits versus $23 \%$ of young rabbits, $p=0.0040$ ) (Figure 1C). Kaplan-Meyer analysis shows aged rabbits had a significantly decreased overall survival post-MI compared to young $(p=0.0285)$, and all mortality of young and aged rabbits occurred within the first 48 hours post-MI (Figure 1D). Of rabbits that initiated peri-procedural VT/VF, we did not find any significant difference in the number or success rate of defibrillation attempts to restore sinus rhythm $(p=0.38$ and $p=0.54$ respectively, data not shown). Overall, our findings indicate the aged rabbit heart is more susceptible to acute, potentially lethal ischemic arrhythmias compared to young rabbits. These trends correlate with the age-associated increase in mortality of out-of-hospital first-time acute $\mathrm{MI}$ in humans [1].

\section{Progression of Infarct Size, IBZ Geometry, and Fibrosis is Consistent Between Young and Aged}

\section{$\underline{\text { Rabbits }}$}

Long-term progressive electrophysiological tissue remodeling at the IBZ can facilitate the initiation and propagation of arrhythmias post-MI even after the ischemic event, increasing risk of SCD [40]. Larger scars and increased IBZ fibrosis can establish a source-sink mismatch and regional heterogeneities in APD and conduction velocity which underlie VT/VF post-MI [41, 42]. Additionally, progressive infarct expansion can 
result in declining cardiac function and increased risk of ventricular wall rupture or VT/VF [43, 44]. However, the cellular mechanisms underlying age-associated differences in post-Ml tissue remodeling and their arrhythmogenic consequences are not well understood. We previously demonstrated that coil embolization of the young and aged rabbit left coronary artery resulted in similar scar size 3 weeks post-MI [10]. Here, we further investigated potential age-associated changes in the dynamics of scar size, shape, and fibrosis throughout a twelve-week timecourse post-MI. Cardiac tissue was harvested from young and aged infarcted rabbits at one, two, and three weeks post-MI to encompass the period following scar formation, and at twelve weeks post-MI to interrogate potential long-term changes.

To assess scar size, we measured the percent infarcted area from epicardial left ventricular free wall dissections via gross anatomic photos. We found no significant difference in the percent infarcted area of the left ventricle between young and aged rabbits at any timepoint (Figure 2A). We then compared the functional area of the IBZ between young and aged infarcted rabbits as represented by protrusions of the scar into the surviving myocardia, since IBZ geometry can affect regional heterogeneities in conduction velocity and APD. We utilized hematoxylin and eosin (H\&E)-stained frozen left ventricle sections from young and aged infarcted rabbit hearts, laid flat along the circumferential-longitudinal plate prior to freezing. To better characterize the 3D structure of the IBZ from each heart, at least 5 slides over at least a $500 \mu m$ endocardial-to-epicardial span per rabbit were analyzed. We found no significant difference in either the number or length of protrusions between young and aged rabbits at any timepoint post-Ml or over time for each age group (Figure 2B). To assess potential age-associated changes in fibrosis post-MI, we analyzed frozen left ventricular sections stained with Masson's Trichrome. We observed no difference at any timepoint between young and aged rabbits post-MI in either percent fibrotic area of the entire left ventricular free wall or in percent interstitial fibrotic area in the remote zone (RZ) (Figure 2C-D). Overall, we observed no significant age-associated differences in the dynamics of scar size, IBZ geometry, or fibrosis in the first twelve weeks post-MI, suggesting the physical properties of the scar are not sufficient to explain age-associated differences in arrhythmogenesis post-MI. 


\section{Electrophysiological Remodeling in the Infarct Border Zone of Aged Rabbit Hearts}

Although aging is associated with changes in numerous biological processes potentially impacting infarct healing, less is known about the role of age-associated changes in post-MI electrophysiological tissue remodeling and how such changes might contribute to differential risk of lethal arrhythmias. We hypothesized more severe electrophysiological remodeling in the aged IBZ compared to young establishes a greater arrhythmic substrate in aged rabbit hearts. To test this hypothesis, we investigated action potential dynamics in young $(n=17)$ and aged $(n=5)$ rabbit hearts ex vivo at 3-weeks post-Ml using optical mapping. The epicardial APDs were recorded using the fluorescent voltage-sensitive dye di-4-ANEPPS and averaged from multiple randomized $4 \mathrm{~cm}^{2}$ regions of the scar, IBZ, and RZ during $350 \mathrm{~ms}$ cycle length stimulation (Figure 3A). Representative action potential traces and APD maps from young and aged rabbit hearts are shown in Figure 3B.

In aged rabbits, APD at the IBZ was significantly longer than in the $R Z(183.1 \pm 6.64 \mathrm{~ms}$ in IBZ and $169.4 \pm 7.90 \mathrm{~ms}$ in $\mathrm{RZ}, \mathrm{p}<0.05)$, whereas in young rabbits, APD in the IBZ was significantly shorter compared to the $R Z(178.9 \pm 6.99 \mathrm{~ms}$ in IBZ and $202.2 \pm 6.32 \mathrm{~ms}$ in $\mathrm{RZ}, \mathrm{p}<0.05)$. Compared to young rabbits, aged rabbits exhibited greater APD heterogeneity along the IBZ (Figure 3B). In both young and aged rabbits, conduction velocity was significantly slower in the IBZ compared to the $R Z(0.32 \pm 0.10 \mathrm{~m} / \mathrm{s}$ in IBZ and $0.96 \pm$ $0.30 \mathrm{~m} / \mathrm{s}$ in RZ of aged hearts, $0.37 \pm 0.05 \mathrm{~m} / \mathrm{s}$ in IBZ and $0.90 \pm 0.13 \mathrm{~m} / \mathrm{s}$ in RZ of young hearts), consistent with previous findings [5]. We observed that the IBZ of aged rabbit hearts can support spatially discordant APD alternans at relatively slow heart rates, which has been associated with an increased risk for arrhythmias (Figure 3C) [45]. These results were not observed in the young hearts under the same conditions. To further study age-associated changes in arrhythmogenic substrate, we induced VF by burst electrical stimulations. Representative VF traces and VF frequency maps are shown in Figure 3D. Aged rabbits exhibited significantly higher dominant VF frequency compared to young, which may be related to the underlying complexity of arrhythmia. Additionally, the aged rabbit hearts displayed greater spatial variation of VF frequencies across the $\mathrm{RZ}$ compared to young, whereas in young rabbits slower-frequency regions tended to cluster near the IBZ. Altogether, these findings suggest that age-associated changes in electrical remodeling 3 weeks post-MI 
increased the heterogeneity of rate-dependent APD dynamic properties between the IBZ and RZ, notably the prolongation in APD in the IBZ of aged rabbits, which demonstrates a more arrhythmogenic substrate.

\section{Senescence of Myofibroblasts is Elevated and Persistent in the Aged Rabbit Heart Post-MI and}

\section{Correlates with Increased Inflammation}

To investigate potential cellular mechanisms underlying the increase in arrhythmogenic substrate in aged infarcted rabbits, we next investigated the dynamics of cellular senescence in young and aged rabbit hearts over time post-MI. Whereas in the proliferative phase post-MI, the scar is rich with myofibroblasts secreting ECM components, in the maturation phase, the fibrogenic activity of myofibroblasts is resolved at least partially via induction of senescence [33,46-49]. To interrogate the dynamics of senescence in young and aged infarcted rabbit hearts, we first performed senescence-associated $\beta$-galactosidase (SA- $\beta$-gal) staining of frozen left ventricular sections from young and aged rabbits over the first twelve weeks post-MI (Figure 4A-C). In the aged rabbit scar, the percent of SA- $\beta$-gal+ cells in the scar remained elevated over time even at 12 weeks post-MI. Conversely in the young rabbit scar, the percent of SA- $\beta-$ gal+ cells was relatively high for the first two weeks and largely resolved by the $3^{\text {rd }}$ week post-MI, resulting in significantly decreased senescence at 3 and 12 weeks post-MI compared to aged rabbits (Figure $4 \mathbf{C}$ ). In the IBZ, the same trend was observed in young and aged rabbits but did not reach statistical significance The young and aged rabbit $\mathrm{RZ}$ displayed very few SA- $\beta$-gal+ cells, consistent with our previous data (not shown)).

To further characterize senescence and determine the cellular identity of senescent cells in the young and aged rabbit scar, we performed immunofluorescence staining on frozen left ventricular sections for the DNA damage marker $\mathrm{yH} 2 \mathrm{AX}$ as a marker of senescence [50] and either the myofibroblast marker aSMA [51] or the endothelial cell marker CD31 [52] (Figure 4D). To account for transient DNA damage in otherwise healthy cells represented by low numbers of $\mathrm{YH} 2 \mathrm{AX}$ nuclear foci, only nuclei exhibiting three or more $\mathrm{YH} 2 \mathrm{AX}$ foci were scored as $\mathrm{yH} 2 \mathrm{AX}$ positive (" $\mathrm{yH} 2 \mathrm{AX}+$ )". These results corroborated our SA- $\beta$-gal data as the percent of $\mathrm{yH} 2 \mathrm{AX}+$ nuclei in the aged rabbits remained elevated over time, whereas the percent of $\mathrm{yH} 2 \mathrm{AX}+$ nuclei in the young rabbit scar was resolved by 3 weeks post-MI, resulting in significant increases in the percent of $\mathrm{YH} 2 \mathrm{AX}+$ nuclei at 3 and 12 weeks post-MI in aged rabbits compared to young (Figure 4E). Specifically, in 
aged rabbits 12 weeks post-MI, we observed $9.9 \pm 3 \%$ of cells were $\mathrm{yH} 2 \mathrm{AX}$ whereas in young rabbits 12 weeks post-MI, only $0.6 \pm 0.2 \%$ of cells were $\mathrm{yH} 2 \mathrm{AX}+$. From our aSMA immunostaining, in both the aged and young rabbit scar we observed initially high percentages of aSMA+ cells followed by a decline over time postMI with no significant difference between young and aged at any timepoint (Figure 4F, left). At 2 weeks postMI, $39.5 \pm 17.5 \%$ of cells were aSMA+ in aged rabbits and $36.5 \pm 8.1 \%$ of cells were aSMA+ in young rabbits. This observation is consistent with previous mouse genetic lineage tracing reports showing fibroblasts that became myofibroblasts remained in the scar but lost aSMA expression by 2 weeks post-MI [46]. From our double immunofluorescence staining, we observed the majority of $\mathrm{yH} 2 \mathrm{AX}+$ cells were aSMA+ at 1-week post$\mathrm{MI}$ in aged and young rabbits, consistent with the high levels of aSMA signal (Figure 4F, right). Interestingly, although at 2 weeks post-MI we observed a $~ 50 \%$ decrease in the percent of aSMA+ cells, we observed that $\sim 80 \%$ of $\mathrm{yH} 2 \mathrm{AX}+$ cells were aSMA+, indicating a propensity towards senescence of myofibroblasts. Specifically, at two weeks post-MI, $66.9 \pm 34.3 \%$ of $\mathrm{yH} 2 \mathrm{AX}+$ cells were aSMA+ in aged rabbits, and $66.2 \pm$ $45.0 \%$ of $\mathrm{yH} 2 \mathrm{AX}+$ cells were aSMA + in young rabbits. At 3- and 12-weeks post-MI, relatively few $\mathrm{YH} 2 \mathrm{AX}+\mathrm{cells}$ were aSMA+, consistent with the decrease in aSMA signal. We observed no significant differences in the percent of $\mathrm{yH} 2 \mathrm{AX}+$ cells that were aSMA+ between young and aged at any timepoint. From our analysis of CD31 immunofluorescence staining, we observed relatively few cells $(\sim 10-20 \%)$ were CD31+ at all timepoints with no significant difference between young and aged rabbits at any timepoint (Figure 4G, left), consistent with previous studies of cell composition post-MI [53]. At the 2-week post-MI timepoint, $12.1 \pm 7.2 \%$ of cells were CD31+ in aged rabbits and $15.8 \pm 11.0 \%$ of cells were CD31+ in young rabbits. Similarly, we observed relatively few $\mathrm{yH} 2 \mathrm{AX}+$ cells were CD31+ across all timepoints with no significant difference between young and aged rabbits at any timepoint, suggesting endothelial cells make up a minor percentage of senescent cells present in the scar post-MI (Figure 4G, right). Two-weeks post-MI, we observed $21.0 \pm 20.1 \%$ of $\mathrm{yH} 2 \mathrm{AX}+\mathrm{cells}$ were $\mathrm{CD} 31+$ in aged rabbits and $2.3 \pm 4.5 \%$ of $\mathrm{yH} 2 \mathrm{AX}+$ cells were CD31+. These findings suggest myofibroblasts make up the majority of persistent senescent cells in the scar of young and aged rabbits.

We next assessed the expression of senescence and SASP transcripts from flash-frozen dissections of the scar, IBZ, and RZ of young and aged rabbits at 3 weeks post-MI. In the aged scar compared to the young scar, we observed a significant upregulation in the expression of all three assayed senescence genes (p16, 
p21, and p53), as well as the SASP components CXCL2, and TIMP2 (Figure 4H). For both young and aged rabbits, in the scar compared to their respective $R Z$, the expression of nearly all assayed senescence and SASP genes was significantly upregulated, except IL-6, MMP9, and TIMP1 for aged rabbits and CXCL2 for young rabbits. In the aged IBZ compared to the young IBZ, we found a significant upregulation in the expression of p16 and p53, as well as a significant decrease in the expression of IL-6 and CCL2. Conversely, in the young and aged IBZ relative to their respective $R Z$, only $p 16$, $p 21$, and TIMP2 were expressed significantly higher in aged and only p16 and TIMP2 were expressed significantly higher in young. In the aged $R Z$ compared to the young $R Z$, we found a significant increase in the expression of p16 and p21, with no significant decrease in expression of any genes. Overall, our data indicate at 3 weeks post-MI, expression of many senescence and SASP factors is upregulated in the aged scar and IBZ compared to young rabbits, consistent with our histological senescence data.

\section{Presence of Senescent Myofibroblasts in Aged Infarcted Human Ventricular Tissue}

To investigate whether the observed elevation and persistence of senescence in the aged rabbit scar is recapitulated in humans, we quantified and characterized senescence in left ventricular cadaveric tissue samples from four aged male human patients with prior MI. The average patient age was $62 \pm 5$ years, three of four patients presented with comorbidities, and heart failure was observed in all patients as represented by the average $2 \mathrm{D}$ ejection fraction of $16 \pm 5 \%$. Tissue samples flash frozen after harvest were embedded in OCT prior to cryosectioning. Representative SA- $\beta-$ gal and Masson's Trichrome-stained frozen sections are shown in Figure 5A. Because very little SA- $\beta$-gal signal was observed, likely due to loss of $\beta$-galactosidase enzymatic activity in the interval between the initial tissue harvest, the flash freezing of tissue, and embedding in OCT, we investigated $\mathrm{yH} 2 \mathrm{AX}$ foci by immunofluorescence to quantify senescence. We performed immunofluorescence staining for $\mathrm{YH} 2 \mathrm{AX}$, aSMA, the endothelial cell marker vWF [52], and the macrophage marker CD68 [54] in the scar. On average, we observed $8 \pm 5 \%$ of total cells were $\mathrm{yH} 2 \mathrm{AX}+$ (Figure $5 \mathrm{~B}-\mathrm{C}$ ), and from our cell identity immunofluorescence analysis we observed an average of $61 \pm 30 \%$ of cells were aSMA+, $17 \pm 12 \%$ of cells were vWF+, and $21 \pm 20 \%$ of cells were CD68+. From our double immunofluorescence staining, we observed an average of $69 \pm 28 \%$ of $\mathrm{yH} 2 \mathrm{AX}+$ cells were aSMA+, $20 \pm 25 \%$ of $\mathrm{yH} 2 \mathrm{AX}+$ cells were vWF+, and $41 \pm 17 \%$ of $\mathrm{yH} 2 \mathrm{AX}+$ cells were CD68+ (Figure 5C). Overall, our analysis of the human scar reveals comparable levels 
of cellular senescence with that seen in the aged rabbits at 3 and 12 weeks post-MI, with around $60 \%$ of senescent cells appearing to be myofibroblasts, similar to observations in the rabbit heart. These findings suggest that senescence-driven pro-arrhythmic remodeling occurring over time in aged infarcted rabbits might also occur in humans.

\section{Establishment of an In Vitro Senescence Model of Adult Rabbit Cardiac Fibroblasts}

To investigate how senescence might promote arrhythmogenesis with age, we first established a method to induce senescence in rabbit primary cardiac fibroblasts in vitro. Primary adult rabbit cardiac fibroblasts were isolated from adult female non-infarcted rabbits, and after 2 passages all cells had homogeneous myofibroblast-like characteristics indicated by immunofluorescence staining positive for aSMA and negative for the endothelial markers CD31 and vWF (Supplemental Figure 1).

Pharmacological induction of senescence is commonly achieved in other cell culture models by treatment with DNA damaging drugs such as etoposide which induce double strand breaks [50, 55]. To determine whether etoposide treatment induces senescence in rabbit cardiac fibroblasts, cells were treated with either vehicle or $10 \mu \mathrm{M}, 20 \mu \mathrm{M}$, or $40 \mu \mathrm{M}$ etoposide and were then stained for SA- $\beta$-gal at various timepoints post-treatment (Figure 6A-B). Untreated, proliferating cells were also stained to assess baseline senescence. An average of $3.9 \pm 1.4 \%$ of untreated, proliferating cells were SA- $\beta-$ gal+, and we found no significant difference in the percent of SA- $\beta-$ gal+ cells in the vehicle group at any timepoint (Figure 6C). For all doses of etoposide, we observed an increase in the frequency of SA- $\beta$-gal+ cells over time. Maximal SA- $\beta$-gal signal at day 12 was $73.9 \pm 13.4 \%$ for the $10 \mu \mathrm{M}$ etoposide group, $86.8 \pm 4.9 \%$ for the $20 \mu \mathrm{M}$ etoposide group, and $85.8 \pm 1.8 \%$ for the $40 \mu \mathrm{M}$ etoposide group.

In addition to SA- $\beta$-gal, we performed immunofluorescence staining against $\mathrm{yH} 2 \mathrm{AX}$ on rabbit cardiac fibroblasts treated with vehicle or $10 \mu \mathrm{M}, 20 \mu \mathrm{M}$, or $40 \mu \mathrm{M}$ etoposide for 6 or 12 days, along with proliferating, untreated cells (Figure 6D) to assess senescence induction. Proliferating cells exhibited on average $1.23 \pm 1.0$ nuclear $\mathrm{YH} 2 \mathrm{AX}$ foci, and we found no significant change in the number of $\mathrm{YH} 2 \mathrm{AX}$ foci per nucleus for vehicletreated cells at any timepoint (Figure 6E). Cells treated with any dose of etoposide exhibited an increase in the number of $\mathrm{yH} 2 \mathrm{AX}$ foci per nucleus over time, reaching a maximum at 12 days of treatment of $19.0 \pm 9.9$ 
YH2AX nuclear foci for the $10 \mu \mathrm{M}$ etoposide group, $10.5 \pm 7.8 \mathrm{yH} 2 \mathrm{AX}$ foci for the $20 \mu \mathrm{M}$ etoposide group, and $16.8 \pm 15.1 \mathrm{\gamma H} 2 \mathrm{AX}$ foci for the $40 \mu \mathrm{M}$ etoposide group. In untreated, proliferating cells, an average of $6.5 \pm$ $7.7 \%$ of cells exhibited more than $5 \mathrm{yH} 2 \mathrm{AX}$ nuclear foci, whereas after 12 days of treatment with $10 \mu \mathrm{M}, 20$ $\mu \mathrm{M}$, and $40 \mu \mathrm{M}$ etoposide, $81.7 \pm 18.3 \%, 59.9 \pm 28.1 \%$, and $64.2 \pm 42.9 \%$ of cells exhibited more than 5 yH2AX nuclear foci (Figure 6F).

Finally, we assessed changes in the expression of senescence and SASP genes in rabbit cardiac fibroblasts treated with vehicle (DMSO alone) or $20 \mu \mathrm{M}$ etoposide over time. We observed no significant difference in transcript levels of any assayed targets between the untreated, proliferating cells and cells treated with vehicle for 6 or 12 days (Figure 6 G). For cells treated with $20 \mu \mathrm{M}$ etoposide for 6 days, we observed a significant increase in the expression of $\mathrm{p} 16$ and $\mathrm{p} 21$. For cells treated with $20 \mu \mathrm{M}$ etoposide for 12 days, we observed a significant increase in the expression of p16, p21, CXCL2, and IL-8. Overall, our data indicate 20 $\mu \mathrm{M}$ etoposide readily induce senescence in the vast majority of rabbit cardiac fibroblasts after twelve days of treatment.

Senescent Adult Rabbit Cardiac Fibroblasts Do Not Appear to Affect Myocyte Electrophysiology in a

\section{Paracrine Manner}

Exogenous treatment of individual proinflammatory factors present in the SASP have been shown to induce electrophysiological dysfunction in myocyte in vitro, and several age-associated diseases are exacerbated by chronic inflammatory signaling via the SASP in other tissues $[35,56]$. We hypothesized that SASP factors from senescent cardiac myofibroblasts in the scar or IBZ may act in a paracrine fashion on IBZ myocytes to alter ion channel function, ultimately prolonging APD and potentially establishing both a substrate and trigger for arrhythmias. To test this hypothesis, we employed an in vitro model using three-week-old rabbit cardiomyocytes and adult rabbit cardiac myofibroblasts [57]. Acutely dissociated cardiomyocytes from 3-weekold rabbits were treated for 30 minutes with conditioned media collected from adult rabbit cardiac fibroblasts treated for 12 days with either vehicle ("Proliferating-CM") or $20 \mu \mathrm{M}$ etoposide ("SASP-CM"), followed by patch clamping of the myocytes. We measured $I_{\mathrm{Kr}}$, the main potassium currents regulating repolarization and APD in these cells, in voltage clamp mode. The holding potential was $-40 \mathrm{mV}$. Three seconds depolarizing voltage steps were applied from $-30 \mathrm{mV}$ to $40 \mathrm{mV}$ in $10 \mathrm{mV}$ intervals. Experiments were done before and after addition 
of $5 \mu \mathrm{M}$ E4031, a specific blocker of $\mathrm{I}_{\mathrm{Kr}}$. The $\mathrm{I}_{\mathrm{Kr}}$ current was determined as E4031 sensitive current (Figure

7A). To generate the $I-V$ curve, $I_{K r}$ was quantified by measuring its tail peak right after the end of the depolarizing pulse (Figure 7B). We observed no significant difference in $\mathrm{I}_{\mathrm{Kr}}$ in myocytes treated with Proliferating-CM versus SASP-CM. We then measured APD in the current-clamp mode in 3-week-old rabbit myocytes treated for 30 minutes with either Proliferating-CM or SASP-CM. Action potentials were evoked at $0.5 \mathrm{~Hz}$ rate, with a $3 \mathrm{~ms}$ depolarizing current pulse to about $30 \%$ above the threshold of the activation of the action potential (Figure 7C). For each cell, the average of at least 20 APD traces were used for quantification. We observed no significant difference between myocytes treated with Proliferating-CM or SASP-CM in either APD50 or APD90, indicating that the conditioned media had no significant effect on the APD. Overall, conditioned media from senescent adult rabbit cardiac fibroblasts does not seem to have a paracrine proarrhythmic effect on 3-week-old rabbit myocytes.

\section{Senescent Cardiac Fibroblasts Can Induce Pro-Arrhythmic Changes in Myocytes via cell-cell}

\section{interactions}

In addition to paracrine effects, senescent cells can act on nearby cells in juxtacrine fashions to induce senescence and cellular dysfunction, both via signaling through cell surface receptors like NOTCH and via direct transfer of intracellular materials via gap junctions [58-60]. This is particularly relevant to cardiac rhythm, as cardiac fibroblasts have been shown to directly couple with myocytes and influence their electrophysiology via gap junctions [61]. Specifically in rabbits, studies have demonstrated functional fibroblast-myocyte gap junction-mediated coupling both via dye transfer assays and via optogenetic assays [38, 61, 62]. To test our hypothesis that senescent myofibroblasts in the IBZ couple with myocytes and promote arrhythmias by altering electrophysiological properties of myocytes, we examined senescence and connexin-43 in frozen cardiac tissue sections from young and aged rabbits 3 weeks post-MI and from adult rabbit cardiac fibroblasts in vitro.

First, we quantified the number of senescent cells in the IBZ that were in immediate proximity to myocytes.

From SA- $\beta$-gal stained frozen sections, we observed a significant increase in the percent of SA- $\beta$-gal+cells that were adjacent to cardiomyocytes in aged rabbits compared to young (Figure $\mathbf{8 A}$ ). Of cells neighboring myocytes, we also observed a significant increase in the average number of SA- $\beta$-gal+ cells per myocyte in 
bioRxiv preprint doi: https://doi.org/10.1101/2021.12.21.473692; this version posted December 23, 2021. The copyright holder for this preprint

(which was not certified by peer review) is the author/funder, who has granted bioRxiv a license to display the preprint in perpetuity. It is made available under aCC-BY-NC-ND 4.0 International license.

aged rabbits compared to young with no difference in the number of SA- $\beta$-gal- cells per myocyte (Figure 8B).

In young rabbits, the average IBZ myocyte had 4 neighboring non-senescent cells and in aged rabbits, the average IBZ myocyte has 4 neighboring non-senescent cells and 1 senescent cell.

To characterize Cx43 expression and localization, we first performed immunofluorescence staining against $\mathrm{Cx} 43$ in proliferating and senescent adult rabbit cardiac fibroblasts. In senescent fibroblasts we frequently observed Cx43 lining the interface between adjacent fibroblasts, implying coupling between the cells (Figure 8C). We next performed immunofluorescence staining of frozen cardiac sections from young and aged rabbits 3 weeks post-MI against Cx43, $\mathrm{HH} 2 \mathrm{AX}$, and aSMA. Particularly in aged rabbits, we observed aSMA+ cells with 3 or more $\mathrm{yH} 2 \mathrm{AX}$ foci immediately near myocytes at the IBZ with $\mathrm{Cx} 43$ appearing at points of contact between the cells, suggesting coupling occurs in vivo (Figure 8D). We observed equivalent results in similarly stained infarcted human ventricular tissue samples, implying the same phenomenon occurs in human cardiac tissue (Figure 8E). Importantly, we did not observe evidence of nuclear $\mathrm{\gamma H} 2 \mathrm{AX}$ foci in myocytes in the IBZ, consistent with our observations in rabbits. In human tissue, the vast majority of apparently coupled cells were aSMA+, and nearly every coupled $\mathrm{yH} 2 \mathrm{AX}+$ cell was also aSMA+.

We next investigated the electrophysiological relevance of potential coupling between senescent cardiac fibroblasts and myocytes by obtaining volumetric and patch clamping data from proliferating and senescent myofibroblasts in vitro and using these data to model the coupling between myocytes and either proliferating or senescent cardiac fibroblasts in silico. We measured the volume of proliferating and senescent cardiac myofibroblasts using confocal microscopy both from frozen sections (Figure 9A, left), and from monolayer cultures (Figure 9A, right) using immunostaining against the lectin wheat germ agglutinin (to delineate the cell surface). In both cases, we observed that senescent myofibroblasts had a significantly larger volume compared to non-senescent ones, consistent with previous literature, and an overall increase in the size of in vitro myofibroblasts compared to those from tissue sections [63]. We next characterized the electrophysiology of single proliferating or senescent adult rabbit cardiac myofibroblasts in vitro via whole-cell patch clamping. The capacitance of proliferating and senescent cardiac fibroblasts in vitro was $128.7 \pm 70.3 \mathrm{pF}$ for proliferating cells and $138.4 \pm 64.0 \mathrm{pF}$ for senescent cells, and along with the volumetric measurements, we extrapolated the capacitance for proliferating and senescent rabbit cardiac fibroblasts in vivo to be $18 \mathrm{pF}$ and 
bioRxiv preprint doi: https://doi.org/10.1101/2021.12.21.473692; this version posted December 23, 2021. The copyright holder for this preprint (which was not certified by peer review) is the author/funder, who has granted bioRxiv a license to display the preprint in perpetuity. It is made available under aCC-BY-NC-ND 4.0 International license.

35 pF (see methods) respectively (Figure 9B). Patch clamping of proliferating and senescent myofibroblasts revealed senescent myofibroblasts to have a slight but nonsignificant increase in peak current compared to proliferating myofibroblasts (Supplemental Figure 2). The voltage-current relationship for each type of cell was fitted with a sigmoidal Boltzmann equation to model the myofibroblast currents.

Using these in vitro measurements, we modeled coupling interactions between single myocytes and either a single proliferating or senescent myofibroblast with a gradient of myofibroblast conductance and capacitance values. Our modeling showed a slight decrease in APD90 with increasing conductance values, and a strong increase in APD90 with increasing capacitance (Figure 9C). With the extrapolated capacitance values for proliferating and senescent myofibroblasts, our modeling suggests myocyte coupling to senescent myofibroblasts results in prolongation of APD. Additionally, we simulated interactions in young tissue (i.e. one myocyte interacting with four non-senescent myofibroblasts) and in aged tissue (i.e. one myocyte interacting with one senescent myofibroblast and four proliferating myofibroblasts), adjusting $\mathrm{Na}^{+}$channel conductance to $50 \%$ of normal values to account for ion channel remodeling observed at the IBZ in canine MI [64]. Simulating myocyte action potentials from young and aged tissue (Figure 9D, first panel), our modeling showed the aged condition resulted in a more prominent notch at phase 1 as well as a prolongation of the APD compared to young (Figure 9D, second panel), consistent with our optical mapping observations of aged rabbits. Our modeling showed that the main cause of APD prolongation in the aged condition is due to the lower plateau $\mathrm{V}_{\mathrm{m}}$ leading to a larger $\mathrm{I}_{\mathrm{Ca}}(-7.4 \mathrm{pA} / \mathrm{pF}$ in the aged vs. $-7.2 \mathrm{pA} / \mathrm{pF}$ in the young condition) (Figure 9D, third panel). The gap junctional currents between myocyte and fibroblasts were greater in the aged condition, $8.1 \mathrm{pA} / \mathrm{pF}$ vs. $7.4 \mathrm{pA} / \mathrm{pF}$ in the young condition (Figure 9D, fourth panel), indicating that myocyte-fibroblast coupling causes the lower plateau $V_{m}$ in the aged condition to increase $I_{C a}$ driving force thereby prolonging APD in the aged condition.

To further investigate potential arrhythmogenic juxtacrine mechanisms of senescent myofibroblasts in silico, we modeled S1S2 protocol in young and aged conditions and investigated at which point premature stimulation of S2 beat fails to induce action potential. Modeling the CL of the S2 beat at which AP failure occurred at variable values for myocyte $\mathrm{Na}$ channel conductance $(\mathrm{gNa}$, normal $=12 \mathrm{mS} / \mu \mathrm{F})$ and fibroblast capacitance $\left(\mathrm{Cm}_{\mathrm{CF}}\right)$ revealed a slight decrease in the $\mathrm{CL}$ at onset of $\mathrm{AP}$ failure with increasing gNa values and 
an increase in the $\mathrm{CL}$ at onset of $\mathrm{AP}$ failure with increasing $\mathrm{Cm}_{\mathrm{CF}}$ (Figure 9E, left). At our extrapolated capacitance values for the young and aged conditions, AP failure occurs at greater CL values under aged conditions compared to young. Representative modeled action potential traces for young and aged simulations during S1S2 protocol are shown in Figure 9E, left. We also modeled the occurrence of AP failure with varying myocyte inward rectifier channel conductance (gK1, normal $\sim 0.30 \mathrm{mS} / \mu \mathrm{F}$ ) values and myofibroblast capacitance values (Figure 9E, right). Compared to our modeling of various gNa values, we observed a steeper relation of increasing gK1 values to decreasing CL values at onset of AP failure for a given capacitance value. Similarly, at the extrapolated values for young and aged capacitance, we found onset of AP failure occurred at higher CL values under aged conditions compared to young when accounting for $\sim 73 \%$ of normal gK1 as experimentally observed in the pig IBZ [64]. These results support that coupling with senescence fibroblasts increases risks for conduction failure, heightening arrhythmogenicity in the aged MI condition.

\section{Discussion}

Although the age-associated accumulation of senescent cells and the related detriments to cardiac function and chronic inflammation have been previously described [65], the relationship between senescence arising during scar formation post-Ml and arrhythmogenesis is not well understood. Our experiments show that accumulation of senescent myofibroblasts in the scar is associated with a tissue microenvironment more susceptible to cardiac arrhythmias and elevated inflammatory signaling. Additionally, our findings suggest senescent myofibroblasts in the IBZ may be exerting pro-arrhythmic effects on surviving myocytes in a cell-cell interaction mediated by myocyte-fibroblast coupling via gap junctions. We propose that persistence of senescent myofibroblasts is a major driver of arrhythmogenic tissue remodeling with age post-MI, and that the timely pharmacologic elimination of senescent cells might alleviate risk of arrhythmias particularly in elderly infarcted patients.

From our MI surgical procedures, we observed significantly increased mortality of aged rabbits mostly due to peri-procedural VT/VF (Figure 1). These results are consistent with the age-associated increase in out- 
of-hospital mortality from cardiac arrhythmias resulting from $\mathrm{Ml}$ [1]. The reason for this increase in periprocedural mortality with age is likely related to our previous observations of increased interstitial fibrosis, disorganization of the His-Purkinje system, and altered myocyte $\mathrm{Ca}^{2+}$ handling with age in non-infarcted aged rabbits, in that these age-associated changes likely increase peri-procedural mortality by lowering the threshold for arrhythmias triggered by the acute ischemic insult $[7,9,66]$. Based on our previous observations showing low levels of senescence in non-infarcted young and aged rabbits (not shown), we posit that senescence likely does not play a role in peri-procedural arrhythmias.

The anatomical characteristics of the scar, including scar size, functional IBZ area, and dense and interstitial fibrosis, could influence age-associated differences in arrhythmogenesis. However, the fact that we did not observe any significant difference in the timecourse of scar size, IBZ area, and overall or interstitial fibrosis suggests that age-associated changes in other cellular processes, including cellular senescence, are driving the increase arrhythmogenicity that we observe from optical mapping studies. Our findings of overall scar size are consistent with our previous study showing $30 \%$ infarcted area of the left ventricle from our MI surgical procedure, and the present data suggest no epicardial infarct expansion occurs over time in young or aged rabbits. [10].

Electrophysiological remodeling at the IBZ includes separation of myocyte bundles via interstitial and replacement fibrosis and significant disruptions of APD and reductions in conduction velocity, myocytemyocyte coupling, and anisotropy ultimately permitting unidirectional conduction block, sustained reentry, and subsequent VT/VF $[4,67,68]$. Although these changes are well characterized in young animal models, we sought to investigate age-associated changes in these processes and their underlying mechanisms. From our optical mapping studies, we observed aged rabbit hearts 3-weeks post-MI exhibited more extreme arrhythmogenic remodeling at the IBZ compared to young, including relative prolongation of APD as compared to the RZ, spatially discordant alternans near the IBZ at relatively slow heart rate, and higher frequency of sustained spontaneous VF following burst pacing. These pathological characteristics indicate aged rabbits undergo remodeling at the IBZ differently than young animals, culminating in a stronger substrate for arrhythmias anchored at the IBZ. Specifically, prolonged APD near the IBZ has been shown to facilitate a reentrant circuit around the scar [69]. Meanwhile, in young rabbits, the significantly shorter APD at the IBZ 
compared to the RZ and scar can also induce and maintain arrhythmia through increasing APD dispersion and action potential conduction block. This finding of shortened APD in the border zone of young rabbits is consistent with previous data using relatively young Yucatan minipigs [64]. However, young infarcted rabbits experience less arrhythmias than aged infarcted rabbits, as indicated by the lack of observed voltage alternans at slow heart rate and slower VF frequency. The observation of similarly slower conduction velocity at the IBZ relative to the remote zone in both young and aged rabbits is consistent with our histological observations showing no difference in the number or length of IBZ protrusions (Figure 2B).

The accumulation of senescent cells in many tissues has been shown to exacerbate age-associated pathologies via paracrine or juxtacrine mechanisms. Studies in mice have demonstrated increased senescence in the scar post-MI with age, the pharmacological clearance of which limits scar size and improves cardiac function and outcome [65]. However, little is known about whether these findings translate to large animal models with more electrophysiological relevance to the human heart, or the mechanisms through which senescent cells might promote arrhythmogenesis in the aged infarcted heart. Here, we show that cellular senescence as measured by SA- $\beta$-gal and $\mathrm{yH} 2 \mathrm{AX}$ staining between young and aged rabbits is similar for the first two weeks post-MI but persists in aged rabbits at three and up to twelve weeks post-Ml in the scar (Figure 4A-E) and is associated with higher local expression of inflammatory SASP factors (Figure 4H). Consistent with previous findings from our group and others [33], we found very few SA- $\beta$-gal positive cells in the RZ of young or aged rabbits, suggesting a low baseline of senescence in the normal young and aged rabbit heart. Additionally, we did not observe any evidence of senescent cardiomyocytes in young or aged rabbits at any timepoint as well as from human ventricular tissues samples from SA- $\beta$-gal staining, which differs from previous reports of mouse tissue [70]. This important observation suggests the rabbit heart could be a more appropriate animal model than the mouse to study the effects of cardiac aging relevant to humans. Interestingly, we observed no difference in either total fibrosis of the LV free wall or interstitial fibrosis over time between young and aged rabbits even though we observed persistence of senescence in the aged rabbit heart over time compared to young. It is well known that the content of the SASP changes over time and depending on cell type and the induction source, and so it is possible that pro-fibrotic and anti-fibrotic SASP factors are present in functionally equivalent amounts ultimately resulting in no difference in fibrosis. This hypothesis is 

available under aCC-BY-NC-ND 4.0 International license.

supported by our RT-qPCR data, indicating upregulation in expression of the pro-fibrotic factors TGF- $\beta$, TIMP1, and TIMP2 as well as anti-fibrotic factor MMP9. Further investigation into this hypothesis is warranted.

From our immunofluorescence staining, the high degree of aSMA signal in young and aged rabbits at 1 and 2 weeks post-MI and the subsequent decrease in aSMA signal is consistent with genetic lineage tracing studies in mice which suggest myofibroblasts remain present in the scar but lose aSMA expression [46]. Our observation that the percent of $\mathrm{yH} 2 \mathrm{AX}+$ cells that are aSMA+ remains high at 2 weeks post-MI when aSMA signal begins to decline suggests that most of the senescent cells present in the scar are aSMA+ myofibroblasts that lose aSMA expression after 2 weeks post-MI. This observation is consistent with previous studies characterizing the identity of senescent cells in the scar post-MI in mice [33, 71$]$. The relatively lower percent of CD31+ cells at any timepoint between young and aged rabbits is consistent with previous reports of cellular composition in the scar [53]. The small percent of $\mathrm{yH} 2 \mathrm{AX}+$ foci that were CD31+ reinforces our conclusion that myofibroblasts are the majority of cells undergoing senescence in the young and aged heart post-MI.

Our RT-qPCR data of young and aged rabbits 3 weeks post-MI further demonstrates differences between the senescence profiles of the aged rabbit and the aged mouse. Consistent with our SA- $\beta$-gal and $\mathrm{yH} 2 \mathrm{AX}$ data, we observed an increase in the expression of $\mathrm{p} 16, \mathrm{p} 21$, and $\mathrm{p} 53$ in aged rabbits compared to young. However, the only SASP factors for which we measured a significant change in expression in the scar zone between young and aged were CXCL2 and TIMP2, whereas the contents of the cardiac SASP were largely different between young and aged mice (albeit with a non-canonical SASP) [70]. Given these results, special attention should be placed on conclusions for the relevance of senescence studies performed in mice in regard to their clinical relevance for humans. Similar to our SA- $\beta$-gal data, we observed a similar but lower magnitude of changes in overall expression between the young and aged rabbit IBZ, and no differences in senescence and SASP gene expression between the young and aged RZ. The significant decrease in IL-6 expression in the aged IBZ compared to young IBZ is interesting, however senescence and SASP signatures are known to be variable depending on cell line and senescence induction method, and this decrease in IL-6 expression has also been observed in studies of young and aged non-infarcted murine myocytes [70]. Overall, our data suggest that senescence and the inflammatory SASP is largely confined to the scar. 
bioRxiv preprint doi: https://doi.org/10.1101/2021.12.21.473692; this version posted December 23, 2021. The copyright holder for this preprint (which was not certified by peer review) is the author/funder, who has granted bioRxiv a license to display the preprint in perpetuity. It is made available under aCC-BY-NC-ND 4.0 International license.

To investigate the clinical relevance of our observations of persistent in the aged infarcted rabbit heart, we measured senescence in aged infarcted human ventricular frozen sections. We observed a relatively high percent of cells in the scar exhibited nuclei with 3 or more $\mathrm{yH} 2 \mathrm{AX}$ foci (Figure $5 \mathbf{C}$ ), indicating the presence of senescent cells in the human tissue, which may contribute to arrhythmogenic tissue remodeling leading to increased incidence of potentially lethal arrhythmias in the chronic phase post-MI. These $\mathrm{yH} 2 \mathrm{AX}$ senescence data are similar to what we observed in the aged rabbit 12 weeks post-MI. From our aSMA analysis of human tissue, we observed a high percent of cells were aSMA+ unlike the progressive decrease in aSMA+ cells over time that we observed in the young and aged rabbits. Because the specific timing of MI is difficult to diagnose especially out-of-hospital, we are unable to ascertain how long post-MI the samples were at time of death. If senescent cells indeed persist in the aged human scar as we hypothesize, it is possible that growth factors like TGF- $\beta$ in the chronic SASP activate fibroblasts in the months-to-years post-MI. Alternatively, the content of the SASP could differ from what we observe in rabbits, contributing to differential activation of myofibroblasts. Similar to our observations in young and aged rabbits, our $\mathrm{yH} 2 \mathrm{AX}$ and aSMA double immunofluorescence assays indicate that the majority of cells with 3 or more $\mathrm{yH} 2 \mathrm{AX}$ nuclear foci were also aSMA+, further suggesting that myofibroblasts are the predominant cell type undergoing senescence. Relatively fewer cells with 3 or more $\mathrm{yH} 2 \mathrm{AX}$ nuclear foci were either vWF+ or CD68+, consistent with previous reports in mice, suggesting relatively fewer senescent cells are endothelial cells or macrophages, respectively [33].

To better study the mechanisms by which senescence might be promoting arrhythmias with age postMI, we established an in vitro method to induce senescence in adult rabbit cardiac fibroblasts. Low doses of the drug etoposide induced senescence, measured by SA- $\beta$-gal and $\gamma \mathrm{H} 2 \mathrm{AX}$ staining as well as RT-qPCR in a time-depending manner, with the vast majority of cells positive for senescence markers after 12 days of treatment with $20 \mu \mathrm{M}$ etoposide (Figure 6). These data establish a platform to future studies screening the efficacy of senolytic drugs to eliminate senescent cells post-MI.

To investigate whether senescent cells might have a paracrine effect on myocytes, we treated primary isolated myocytes from 3-week-old rabbits with conditioned media harvested from proliferating (Proliferating$\mathrm{CM}$ ) or senescent (SASP-CM) fibroblasts (Figure 7). From our patch clamping studies, we observed no significant difference in $\mathrm{I}_{\mathrm{Kr}}$ current or in APD between Proliferating-CM or SASP-CM groups. These findings 
suggest that senescent rabbit fibroblasts likely do not act in a paracrine manner to disrupt the

electrophysiology of rabbit myocytes, but further study is needed to verify this. Because we are limited by the number of commercially-available assays to determine the specific contents of the rabbit SASP, we are unable to exclude the possibility that the conditioned media lacked sufficient levels of SASP factors to induce a significant effect, that conditioned media duration was insufficient, or that degradation of conditioned media occurred between harvest and treatment, as well as potential issues with incubation period and responsiveness of the 3-week-old myocytes to the SASP. We next investigated whether senescent fibroblasts might directly couple with myocytes at the IBZ to influence pathological electrophysiological remodeling. Homoand hetero-typic coupling occurs in the healthy and diseased heart via connexins, including Cx43 [72]. We observed a significantly higher percentage of senescent cells neighboring IBZ myocytes in aged rabbits 3weeks post-MI compared to young. Our immunofluorescence studies suggest Cx43 facilitates homocellular coupling between senescent adult rabbit cardiac fibroblasts, as well as heterocellular coupling between senescent myofibroblasts and myocytes at the IBZ of aged rabbits and human frozen sections.

Our computational modeling of proliferating and senescent myofibroblasts as well as simulations of cardiomyocyte-myofibroblast interactions in young and aged tissue shed critical light for explaining mechanisms by which senescent myofibroblasts might promote arrhythmogenic tissue remodeling with age post-MI. Although electrical remodeling in the IBZ is a complex phenomenon with multiple contributing factors, our results indicate that myocyte-fibroblast interaction becomes a potential contributor to arrhythmogenesis in the aged MI condition. A large, flattened morphology is one of the hallmark characteristics of senescent cells in other models [73], and we observed this for cardiac myofibroblasts both in vitro and in vivo. The larger size of senescent myofibroblasts likely explains the greater extrapolated values for in vivo capacitance, and all differences in modeling between proliferating and senescent myofibroblasts are driven by the larger capacitance of senescent myofibroblasts. Importantly, the prolongation in APD from our modeling of aged condition relative to young is consistent with our optical mapping findings from young and aged rabbits 3 weeks post-MI and validates the relevance of our modeling. From our S1S2 modeling under young and aged conditions, we would predict that aged rabbits would have higher risks for conduction block than young rabbits. Although we did not observe a significant difference in the CL at which VF was induced, VF frequencies were 
higher and heterogeneous in the infarct border zone (Fig. 3D), suggesting that the prolongation of APD in myocytes coupled to senescent myofibroblasts would result in lower threshold for conduction block and wavebreak, overall driving faster VF frequency. These observations are consistent with our hypothesis that myocyte coupling to senescent myofibroblasts in the IBZ of aged rabbits leads to proarrhythmic electrophysiological remodeling and increases risk of reentrant current anchored to the IBZ.

In summary, we have characterized an age-associated accumulation of senescent myofibroblasts in the aged infarcted rabbit heart, and our findings suggest that these senescent cells remodel the electrophysiology of IBZ myocytes at least via direct myocyte-fibroblast coupling mediated by Cx43, which would increase risk of cardiac arrhythmias. Previous studies have shown that pharmacological elimination of senescent cells with age via the senolytic drug navitoclax limits cardiac fibrosis in infarcted and non-infarcted mice and improves the survival and cardiac function of infarcted young and aged mice, but little is known about the effects of senescence on arrhythmogenesis with age $[65,70]$. Our findings demonstrate an important role of persistent senescence in arrhythmogenesis with age post-MI, suggesting the use of senolytic therapy to mitigate senescence burden with age might be promising strategy to mitigate chronic arrhythmias in aged infarcted individuals.

\section{Methods}

Animal Ethical Statement. This investigation conformed with the current Guide for Care and Use of Laboratory Animals published by the National Institutes of Health (NIH Publication, Revised 2011) as well as the standards recently delineated by the American Physiological Society ("Guiding Principles for Research Involving Animals and Human Beings") and was approved by the Institutional Animal Care and Use Committee of Rhode Island Hospital. Young ( $\leq 1$ year old) and aged ( $\geq 4$ years old) female New Zealand White rabbits were infarcted as previously described $[10,11]$. Rabbits received either a peri-procedural 5mg/kg/hr amiodarone infusion followed by a single $400 \mathrm{mg}$ oral dose of amiodarone one day post-MI, or a peri-procedural $1 \mathrm{mg} / \mathrm{kg} / \mathrm{hr}$ amiodarone infusion followed by $120 \mu \mathrm{g} / \mathrm{kg} / \mathrm{hr}$ IV amiodarone continually for three days post-MI delivered by iPRECIO pump (Data Sciences International, St. Paul, MN) implanted into the right jugular vein. 

available under aCC-BY-NC-ND 4.0 International license.

Tissues. Rabbits were anesthetized with ketamine $(60 \mathrm{mg} / \mathrm{kg})$, xylazine $(15 \mathrm{mg} / \mathrm{kg} \mathrm{IM})$, buprenorphine $(0.03$ $\mathrm{mg} / \mathrm{kg} \mathrm{SQ}$ ), and sodium pentobarbital (150 mg/kg IV). Hearts were excised as previously described [10] and washed in PBS. Gross anatomy photos of the intact LV were taken immediately after beating heart harvest to assess percent scar size of the LV free wall in Adobe Photoshop by freehand selection of the scar and the LV free wall; analysis for each heart was performed by three blinded individuals. Heart tissue for histological analysis was embedded in optimal cutting temperature (OCT) compound solution (Fisher Scientific, Waltham, MA, USA) and rapidly frozen on liquid nitrogen. Heart tissue for biochemical analysis was snap frozen in liquid nitrogen and stored immediately at $-80^{\circ} \mathrm{C}$. Cryosectioning was performed at $6 \mu \mathrm{m}$ thickness using a Leica CM1950, and slides were immediately fixed in cold 4\% PFA in PBS for 15 minutes followed by a wash in PBS for 15 minutes, with the exception of slides with Cx43 staining, in which slides were fixed in cold acetone for 10 minutes.

Interdigitation analysis was performed on cryosections stained with Hematoxylin and Eosin as previously described [74] and imaged using an Aperio ScanScope (Leica Biosystems, Buffalo Grove, IL, USA) and analyzed using Aperio ImageScope v12 software (Leica Biosystems, Buffalo Grove, IL, USA). The freehand pen tool was used to mark a continuous IBZ, and the ruler tool was used to determine the number and length of interdigitations protruding from the continuous IBZ marker into the myocardia. All interdigitation analysis was performed by at least three blinded individuals, and multiple cryosections per heart were analyzed.

Optical Mapping of Epicardial Surface and Data Analysis. Young and aged hearts were prepared as described previously $[45,75]$. Hearts were stained with the voltage-sensitive dye di-4-ANEPPS, and ECG and perfusion pressure were constantly monitored (PowerLab, ADInstruments, Sydney, Australia). A total of 5 young and 18 aged rabbits were used. The imaging apparatus has been previously described [76]. The data were analyzed with a custom-built software program developed in Interactive Data Language (Harris Geospatial Solutions). Action potential durations and APD maps were calculated via the difference between activation and repolarization timepoints, determined from fluorescence $(F)$ signals by calculating $(d F / d t)_{\max }$ and $\left(d^{2} F / d t^{2}\right)_{\max }$. Data were filtered using a temporal polynomial filter ( $3^{\text {rd }}$ order, 13 points) [77]. Action potential conduction 
velocity was calculated using the spatial gradient of activation time $(11 \times 11$ pixels $)$ and spatial resolution of acquired image $(200 \mu \mathrm{m} /$ pixel).

Stimulation Protocol. The atrioventricular (AV) node was ablated to control heart rate using a cautery unit (World Precision Instruments, Sarasota, FL, USA). After ablation, the rabbits exhibited a slower heart rate, with the cycle length typically ranging from $700 \mathrm{~ms}$ to $2000 \mathrm{~ms}$. If the cycle length was longer than $2000 \mathrm{ms,} \mathrm{the}$ base of the right ventricle was paced at a cycle length of $2000 \mathrm{~ms}$. Ramp pacing protocol: Hearts were paced at CLs that were successively decreased by $10 \mathrm{~ms}$ until loss of $1: 1$ capture or induction of ventricular fibrillation or ventricular tachycardia [78-80].

SA- $\beta$-Galactosidase Assay: Frozen Tissue. SA- $\beta$-Gal staining was performed with modifications as previously described [81, 82]. Tissue sections immediately after cryosectioning were immersed in $0.5 \%$ glutaraldehyde (in $1 \mathrm{X}$ PBS) for 15 minutes then placed in fresh $1 \times$ PBS. Sections were then incubated at $37^{\circ} \mathrm{C}$ with no $\mathrm{CO}_{2}$ with fresh $\beta$-galactosidase staining solution ( $40 \mathrm{mM}$ citric acid/ $\mathrm{Na}$ phosphate buffer, $5 \mathrm{mM} \mathrm{K} \mathrm{K}_{4}\left[\mathrm{Fe}(\mathrm{CN})_{6}\right] \cdot 3 \mathrm{H}_{2} \mathrm{O}, 5 \mathrm{mM}$ $\mathrm{K}_{3}\left[\mathrm{Fe}(\mathrm{CN})_{6}\right], 150 \mathrm{mM}$ sodium chloride, $2 \mathrm{mM}$ magnesium chloride, and $1 \mathrm{mg} \cdot \mathrm{ml}-1 \mathrm{X}$-gal) for 16 hours. Sections were counterstained with Hematoxylin and Eosin, and slides were imaged using an Aperio ScanScope (Leica Biosystems, Buffalo Grove, IL, USA) with a 40x Objective. Images were analyzed using Aperio ImageScope v12 software (Leica Biosystems, Buffalo Grove, IL, USA). The percent of SA- $\beta$-Gal positive cells was calculated by hand counting the number of SA- $\beta-$ Gal positive and SA- $\beta-$ Gal negative cells from at least 5 randomly chosen images, using the counter tool in ImageJ, by at least three individuals.

Cell Culture Measurements. SA- $\beta-$ Gal staining of rabbit cardiac fibroblasts in cell culture plates was performed as previously described, in triplicate [83]. Cells were imaged in PBS at using a Nikon Eclipse TE2000U with a Retiga EXI color camera. Images were acquired with a 20X FLUOTAR NA Objective under differential interference contrast (DIC). At least five images were acquired per biological replicate. The percentage of SA$\beta$-Gal positive cells were calculated by hand in ImageJ as described above.

Immunofluorescence Staining: Frozen Tissue. Frozen sections were stained via immunofluorescence with some modifications as previously described [84]. Briefly, $6 \mu \mathrm{m}$-thick frozen sections were acquired and fixed as described above. Tissue was encircled with a PAP pen. For intracellular targets, frozen sections were 
bioRxiv preprint doi: https://doi.org/10.1101/2021.12.21.473692; this version posted December 23, 2021. The copyright holder for this preprint (which was not certified by peer review) is the author/funder, who has granted bioRxiv a license to display the preprint in perpetuity. It is made available under aCC-BY-NC-ND 4.0 International license.

permeabilized with $0.1 \%$ Triton X-100 in PBS for 30 min. Slides were then washed with PBS and blocked with $3 \%$ bovine serum albumin, normal donkey serum, or normal goat serum in PBS for $1 \mathrm{hr}$. Slides were then incubated with primary antibody diluted in blocking buffer overnight at $4^{\circ} \mathrm{C}$ in the dark, then washed in PBS, and incubated in secondary antibody in blocking buffer for $2 \mathrm{hr}$ in the dark. Slides were then mounted with Prolong Gold Reagent with DAPI and imaged with a Nikon Ti2 confocal microscope with an A1R scanner. At least 5 images with at least 10 Z-stacks were acquired randomly for each sample. Antibodies used are listed in

\section{Supplemental Table 1.}

The number and percentage of cells with $\mathrm{yH} 2 \mathrm{AX}$ and aSMA signal were derived from maximum projection images by hand using the counter tool in ImageJ. All measurements were performed by two blinded individuals. A $\mathrm{yH} 2 \mathrm{AX}$ nuclear focus was identified if at least one distinct focus overlapped the DAPI-positive nucleus; large, continuous regions of signal in the $\mathrm{yH} 2 \mathrm{AX}$ channel inside a nucleus and any signal outside the nucleus were considered artifact and were excluded from analysis. A cell was considered aSMA-positive if signal in the aSMA channel was present within $10 \mu \mathrm{m}$ of a nucleus and displayed the expected morphology.

Cell Culture. Cells in triplicate were fixed in wells with 4\% PFA for 15 minutes, then washed with PBS. Cells were incubated with blocking solution ( $5 \%$ normal serum corresponding to host of secondary antibody, $0.3 \%$ Triton X-100 in 1X PBS) for $1 \mathrm{hr}$. Cells were then treated with primary antibody diluted in blocking solution for $16 \mathrm{hr}$ at $4^{\circ} \mathrm{C}$, then washed in PBS. Cells were then incubated in secondary antibody diluted in blocking solution for $2 \mathrm{hr}$ in the dark, washed in PBS, then mounted with Prolong Gold Reagent DAPI and imaged with a Nikon Eclipse TE2000U inverted microscope at 40X. Multiple Z-stack images with $0.5 \mu \mathrm{m}$ step length were taken per view, and at least five views per replicate were imaged. To computationally calculate the number of $\mathrm{yH} 2 \mathrm{AX}$ foci per nucleus, a maximum intensity projection was created for each Z-stacked image and individual nuclei were cropped in ImageJ, and a custom analysis macro was run (Supplemental File 1).

RT-qPCR: Tissue. Total RNA was harvested from 40-50 mg of LV tissue (scar zone, infarct border zone, and remote zone) using Trizol reagent (Invitrogen). Nucleic acid concentration of the RNA was determined using a Nanodrop 2000c Spectrophotometer (ThermoFisher, Waltham, MA, USA). Reverse transcription was performed using the iScript reverse transcription kit (BioRad, Hercules, CA, USA) and a C1000 Thermocycler (BioRad, Hercules, CA, USA). Primer sets were designed for each gene of interest as well as endogenous 
control. National Center for Biotechnology Information gene database (https://www.ncbi.nlm.nih.gov/gene) was used to obtain accession numbers for each gene, and Primer Blast tool

(https://www.ncbi.nlm.nih.gov/tools/primer-blast/) was used to yield several primer sets for each gene. Primer sets were selected for each gene of interest for subsequent testing. The efficiencies of the selected primer set for each gene were determined (Supplemental Table 2) to accurately calculate normalized change gene expression $(\Delta \mathrm{CT})$. Real-time qPCR was performed using SYBR Green chemistry (BioRad) and a ViiA 7 Real Time System (ThermoFisher, Waltham, MA, USA). Primer efficiency was calculated using $40 \mathrm{ng}, 4 \mathrm{ng}, 0.4 \mathrm{ng}$, $0.04 \mathrm{ng}$, and $0.004 \mathrm{ng}$ of cDNA from rabbit LV free wall tissue performed in triplicate for each primer set. SRP14 internal control [85] and no template were used as positive and negative controls, respectively. Standard curves, melt curves, and primer efficiency were reported by the ViiA7 QuantStudio software.

RT-qPCR: In vitro. Cells plated in $10 \mathrm{~cm}$ plastic uncoated dishes (CytoOne, USA Scientific) were harvested with Trizol reagent (Invitrogen). Nucleic acid concentration of the RNA was determined using a Nanodrop 2000c Spectrophotometer (ThermoFisher, Waltham, MA, USA). Reverse transcription was performed using the iScript reverse transcription kit (BioRad, Hercules, CA, USA) and a C1000 Thermocycler (BioRad, Hercules, CA, USA). Real-time qPCR was performed using SYBR Green (BIO-RAD and a ViiA 7 Real Time System (ThermoFisher, Waltham, MA, USA) as specified by the manufacturer. Primers used are listed in

Supplemental Table 2 For each condition, senescence of cells plated and treated in parallel was confirmed visually via SA- $\beta$-gal staining: all vehicle treated cells showed low percent of SA- $\beta$-gal+ cells and all etoposidetreated cells showed a high degree of SA- $\beta-g a l+$ cells.

Adult Rabbit Cardiac Fibroblast Isolation and Culturing. Primary adult rabbit cardiac fibroblasts (rabbit cardiac fibroblasts) were isolated from aged ( $>4$ years) New Zealand White rabbits with modifications from previously described [8], by Langendorff perfusion with a solution containing collagenase II (\#CLS-2, Worthington Biochemical, Lakewood, NJ, United States). After isolation, instead of using the myocyte-containing pellet, the supernatant was centrifuged three times at $400 \mathrm{rpm}$ for 5 minutes, transferring the supernatant to a new tube each time. After the third centrifugation, the supernatant was aspirated and the pellet was resuspended in rabbit fibroblast media (DMEM/F-12 (Life Technologies) supplemented with $10 \%$ fetal bovine serum, penicillin (100 $\mu / \mathrm{mL})$, and streptomycin $(0.1 \mu / \mathrm{mL})$ (\#P4333, Sigma, St. Louis, MO, United States). Cells were plated 
onto $10 \mathrm{~cm}$ plastic dishes (CytoOne, USA Scientific) and grown for three passages in a $37^{\circ} \mathrm{C}$ incubator at atmospheric oxygen before cell identity immunofluorescence staining (Supplemental Figure 1) and subsequent experiments. Passaging was performed using $0.05 \%$ Trypsin-EDTA (Thermo Fisher) for a maximum of 4 min.

Rabbit cardiac fibroblasts were plated onto 24-well uncoated plates (CytoOne, USA Scientific). Once they reached $\sim 30 \%$ confluence, rabbit cardiac fibroblasts were treated with rabbit fibroblast media supplemented with either dimethylsulfoxide (DMSO, Thermo Fisher) vehicle or Etoposide (MedChemExpress), replacing with fresh media and drug every 2-3 days. For conditioned media, cells were washed in PBS before addition of 3wkRCM media for $24 \mathrm{hr}$, after which conditioned media was harvested, centrifuged at 1000x $\mathrm{g}$ for $5 \mathrm{~min}$ at $4^{\circ} \mathrm{C}$, then filtered with a $0.45 \mu \mathrm{m}$ cell filter(CytoOne). Cells plated and treated in parallel were used to count cells, and aliquots of equal cell density were stored at $-80^{\circ} \mathrm{C}$. For use, conditioned media was thawed in a $37^{\circ} \mathrm{C}$ water bath, diluted $1: 1$ with fresh $3 w k R C M$ media, then immediately treated onto $3 w k R C M s$.

3-week-old Rabbit Cardiomyocyte Isolation. Primary cardiomyocytes were isolated from the LV and septum of 3-week-old female rabbits as previously described [8, 57]. Myocytes were incubated with either SASP-CM, Pro-CM or UCM for 30 minutes in a $37^{\circ} \mathrm{C}$ incubator before patch clamping.

Electrophysiology Recording. Whole-cell patch clamp recordings from proliferating and senescent fibroblasts were performed with an Axopatch-200B amplifier and pCLAMP 10 software (Molecular Devices, San Jose, CA) at $34-36{ }^{\circ} \mathrm{C}$. Capacitance and 60 to $80 \%$ of series resistance were routinely compensated. The sampling frequency was $20 \mathrm{kHz}$, and $-3 \mathrm{~dB}$ cut-off frequency was $5 \mathrm{kHz}$. The pipette solution contained $110 \mathrm{mM} \mathrm{K}$ aspartate, $20 \mathrm{mM} \mathrm{KCl,} 1 \mathrm{mM} \mathrm{MgCl}$, 0.05mM EGTA, $10 \mathrm{mM}$ HEPES, 5 mM K ${ }_{2}$ ATP, $0.1 \mathrm{mM}$ Tris-GTP, and 5 $\mathrm{mM} \mathrm{Na}_{2}$-phosphocreatine $(\mathrm{pH} 7.3$ with $\mathrm{KOH})$. Tyrode solution was used as a bath solution and contained: 140 $\mathrm{mM} \mathrm{NaCl}, 5.4 \mathrm{mM} \mathrm{KCl}, 1.8 \mathrm{mM} \mathrm{CaCl}_{2}, 1 \mathrm{mM} \mathrm{MgCl}_{2}, 0.33 \mathrm{mM} \mathrm{NaH}_{2} \mathrm{PO}_{4}, 5.5 \mathrm{mM}$ glucose, and $10 \mathrm{mM}$ HEPES $(\mathrm{pH} 7.3$ with $\mathrm{NaOH})$. Fibroblasts were clamped at $-60 \mathrm{mV}$ holding potential followed by a series of 3 second voltage steps from $-100 \mathrm{mV}$ to $40 \mathrm{mV}$. To minimize changes in the intracellular ion concentrations in the fibroblasts, the resting potential was recorded with sharp electrodes (30-40 M $\Omega$ ) filled with $3 \mathrm{M} \mathrm{KCl}$ in the current-clamp mode $(I=0)$ of the amplifier. 
Rabbit Ventricular Myocyte Action Potential and Fibroblast Modeling. We used the rabbit ventricular myocyte

AP model of Mahajan et al. [86] coupled to a passive current fibroblast model based on the currents we recorded in patch clamp experiments. The current amplitudes were measured relative to the tail of the current at the end of the voltage step to minimize the inclusion of leak current. The resulting normalized currentvoltage relation was plotted for proliferating and senescent fibroblasts and each fit with a Boltzmann equation (see Supplementary Figure 2). The Boltzmann curves were shifted to bring the reversal potential to the resting potentials recorded with sharp intracellular electrodes. The resulting equations were used to model the proliferating and senescent fibroblast currents with the addition of gPro and gSen conductance constants multiplied by the equations to allow adjustment of the current amplitude. To calculate the extrapolated capacitance for the proliferating and senescent myofibroblasts used in the model, the surface area was calculated from the mean volumes in Figure 9A assuming spherical cells. Then we calculated the ratio of a single fibroblast surface area in tissue to a single fibroblast surface area in culture for proliferating and senescent cells. These ratios were used to scale the capacitance values measured by whole-cell patch clamp (Figure 9B) to "tissue" capacitance values. Since the measured capacitance was similar in proliferating and senescent fibroblasts (probably due to the normalization of cell size with trypsin treatment on the day of recording), we used the tissue-extrapolated capacitance value for proliferating cells and then multiplied that by 1.95 , the ratio of senescent to proliferating fibroblast surface area in tissue. The gap junction conductance was set to $20 \mathrm{nS}$. Numerical calculation of the different equations in the model were performed by the Euler method with an adaptive time step of $t=0.05 \sim 5 \mu$ s. The simulations to investigate the effect of coupling senescent myofibroblasts to ventricular myocytes were carried out with double precision on a Nvidia Tesla K40 multicore graphic processing unit using the CUDA toolkit (https://developer.nvidia.com/cuda-zone). The data visualization was done using Python.

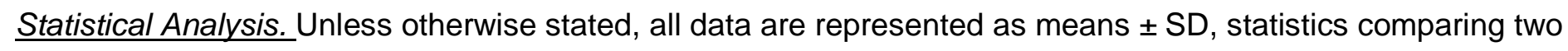
groups were two-tailed Exact tests, and statistics comparing more than two groups were two-way ANOVA with Bonferroni correction. GraphPad Prism 9.1 was used to perform all statistics and create all graphs. 


\section{Author Contributions}

BCB generated majority of the data, performed data analysis, and wrote the first draft of the article. KRM generated the rabbit qPCR data and generated data contributing to Figures 1 and 4. ES performed immunofluorescence staining troubleshooting assisting data generated in Figure 4. EM generated data contributing to Figure 2. YC generated data contributing to Figure 4. NT and LS performed rabbit surgeries and harvests. TYK and BRC generated and analyzed optical mapping data. AYK and PB generated and analyzed patch clamping data. PB and BRC performed computational modeling, with input from ZQ. PC provided critical input in the cell-cell interaction assay design. PD provided critical input regarding inflammation studies. DT provided critical input in the conditioned media and patch clamping experiments. FDM provided human tissue samples. JS provided critical input in in vitro experiments and overall experimental design. GK supervised all experiments and writing of the article.

\section{Acknowledgements}

We thank Dr. Alison Chambers for her consultation on biostatistics. We also thank Dr. Christoph Schorl at the Genomics Core of Brown University for his assistance with qPCR hardware and bioanalysis of RNA samples. We thank Dr. Patrycja Dubielecka for her consultation regarding markers of inflammation. We also thank Cindy Phun, Maria Veliz, Tiffany Borjeson, and the rest of the Rhode Island Hospital Central Research Facilities for assistance during rabbit surgeries and coordination and care of rabbits before and after surgery. 


\section{Sources of Funding}

This work was funded by NIH grants R01HL139467 and 1R1AG049608-01

\section{Figure Legends}

Figure 1: Aged Rabbits Exhibit Increased Incidence of Peri-Procedural Arrhythmias

(A) Survival table of young and aged infarcted rabbits. Procedural deaths were defined as death occurring within the first 3 hours of surgery. (B) Histogram of rabbits included in the study by age at the time of surgery (C) Incidence of procedural VT/VF in young and aged infarcted rabbits. Numbers inside bars are number of rabbits. * $p<0.05$, two-tailed Exact test. (D) Survival curves of young and aged rabbits post-MI $(p<0.05$, Log Rank test).

Figure 2: Progression of Infarct Size, IBZ Geometry, and Fibrosis is Consistent Between Young and Aged Rabbits

(A) Left: Whole rabbit heart, with infarct zone outlined. Middle: Dissected left ventricular free wall. Right: Quantification of percent infarcted area of the left ventricular free wall. (B) Left: representative H\&E-stained images of infarct bord zone (left) protrusions into surviving myocardia (right). Right: quantification of number (top) and length (bottom) of protrusions. (C) Left: Representative Masson's Trichrome stained left ventricular section. Right: Quantification of percent fibrotic area. (D) Left: Representative Masson's trichrome stained remote zone images showing interstitial fibrosis. Right: quantification of \% fibrotic interstitial area.

Figure 3: The Border Zone of Aged Rabbits Shows APD Prolongation, APD Alternans, and Faster VF Frequency

(A) Photograph of a rabbit heart showing the infarct zone (IZ), infarct border zone (IBZ), and remote zone (RZ).

(B) Top: Representative AP traces from the RZ and IBZ of young and aged rabbits. Middle: Representative 
APD maps from young and aged rabbits, showing APD at different points along the border zone. Bottom:

Quantification of APD and conduction velocity in the RZ and IBZ of young and aged rabbits. * $p<0.05$, two-

tailed Exact test. (C) Representative AP trace of an aged infarcted rabbit at 3 weeks post-MI showing alternans

in the IBZ. (D) Top: Representative traces showing VF in young and aged rabbits after electrical induction.

Bottom: Representative VF frequency maps of IBZ of young and aged rabbits. Right: Quantification of VF

frequency and cycle length at which VF was induced from young and aged rabbits. * $p<0.05$, two-tailed Exact

test. Dots represent average data for each rabbit.

\section{Figure 4: Senescence of Myofibroblasts is Elevated and Persistent in the Aged Rabbit Heart Post-MI}

\section{and Correlates With Increased Inflammation}

(A) Representative SA- $\beta$-gal-stained image showing examples of infarct zone, border zone, and remote zone.

(B) Representative SA- $\beta$-gal-stained images showing infarct zone, border zone, and remote zone from young and aged rabbits at 3 weeks post-MI. (C) Quantification of percent SA- $\beta$-gal+ cells from the scar (top), IBZ (middle), and RZ (bottom) in young and aged rabbits at 1, 2, 3, and 12 weeks post-MI. (D) Representative confocal images of aSMA/ $\mathrm{YH} 2 \mathrm{AX}$ double immunofluorescence staining (top row) and CD31/ $\mathrm{YH} 2 \mathrm{AX}$ double immunofluorescence staining (bottom row) from young (left) and aged (right) in the infarct zone of rabbits at 12 weeks post-MI. White indicates autofluorescence and was used to avoid false positive fluorescence signal. (E) Quantification of \% of nuclei with 3 or more $\mathrm{yH} 2 \mathrm{AX}$ foci. (F) Quantification of percent aSMA+ cells (left) and the percent of $\mathrm{yH} 2 \mathrm{AX}+$ cells that are aSMA+ (right). (G) Quantification of percent CD31+ cells (left) and the percent of $\mathrm{yH} 2 \mathrm{AX}+$ cells that are CD31+ (right). (H) Quantification of expression of senescence and SASP genes via RT-qPCR from young and aged rabbits 3 weeks post-MI. * $\mathrm{p}<0.05$ compared to young, $\mathrm{N}=3$ rabbits per condition.

\section{Figure 5: Presence Of Senescent Myofibroblasts In Aged Infarcted Human Ventricular Tissue}

(A) Representative images of SA- $\beta$-gal (left) and Trichrome (right) stained frozen sections. (B) Representative confocal images of $\mathrm{yH} 2 \mathrm{AX} / \mathrm{aSMA}$ double immunostained frozen sections. Yellow arrows indicate nuclear 
yH2AX foci. (C) Quantification of \% $\mathrm{yH} 2 \mathrm{AX}+$ cells (left), cell identity markers (middle), and \% of $\mathrm{yH} 2 \mathrm{AX}+$ positive for cell identity markers (right) from B).

\section{Supplemental Figure 1: Verification of Adult Rabbit Cardiac Fibroblast Identity}

Representative images from immunofluorescent staining of adult rabbit cardiac fibroblasts (top) and human aortic endothelial cells (bottom) with myofibroblast and endothelial cell markers

Figure 6: Etoposide Induces Senescence in Adult Rabbit Cardiac Fibroblasts

(A) Experimental design. (B) Representative SA- $\beta$-gal stained images of treated fibroblasts. (C) Quantification of \% SA- $\beta$-gal+ cells from B). (D) Representative nuclear $\mathrm{\gamma H} 2 \mathrm{AX}$ stained images of treated fibroblasts. (E) Quantification of \# yH2AX foci from D). (F) Quantification of distribution of $\mathrm{yH} 2 \mathrm{AX}$ foci from $D)$. (G) Quantification of RT-qPCR of treated fibroblasts

Figure 7: Conditioned Media from Senescent Adult Rabbit Cardiac Fibroblasts Does Not Affect APD or $\mathbf{I}_{\mathbf{K r}}$

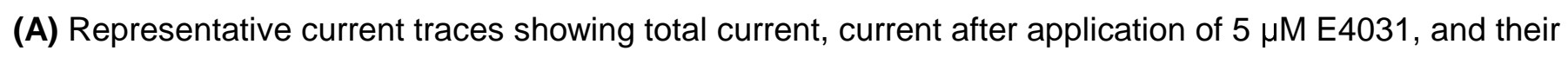
difference that reveals $I_{\mathrm{Kr}}$ of primary 3-week-old rabbit myocytes treated with conditioned media from adult rabbit cardiac fibroblast. Peak of the tail of $\mathrm{I}_{\mathrm{kr}}$ was measured at different potentials. Insert shows voltage protocol. (B) Cumulative data of $\mathrm{I}_{\mathrm{kr}}$ current. Numbers in parenthesis are number of cells analyzed. (C) Representative APD traces from primary 3 week old rabbit myocytes treated with conditioned media from adult rabbit cardiac fibroblasts. (D) Quantification of C).

Figure 8: Pro-Arrhythmic Effect of Myocytes Coupled to Senescent Fibroblasts Compared to NonSenescent Fibroblasts 
(A) Quantification of the percent of SA- $\beta$-gal+ cells adjacent to myocytes (left) and representative SA- $\beta$-gal stained images of the IBZ from young and aged rabbits 3 weeks post-MI. (B) Quantification of the number of adjacent SA- $\beta$-gal+ cells per myocyte (left) and the number of adjacent SA- $\beta$-gal- cells per myocyte (right) from the young and aged rabbit IBZ 3 weeks post-MI. (C) Representative confocal image of senescent adult rabbit cardiac fibroblast immunofluorescent staining against Cx43. Line of Cx43 between cells indicated with white arrows. (D) Representative confocal images of immunofluorescent staining against Cx43, yH2AX, and aSMA of the IBZ of young and aged rabbits 3 weeks post-MI. Yellow arrows indicate nuclei with 3 or more nuclear yH2AX foci. (E) Representative confocal image (left) of immunofluorescent staining against Cx43, yH2AX, aSMA, and Troponin T of the IBZ of aged human IBZ samples and quantification of \% of coupled cells (right). Yellow arrows indicate nuclei with 3 or more nuclear $\mathrm{YH} 2 \mathrm{AX}$ foci.

\section{Figure 9: Computational Modeling of Myocyte-Myofibroblast Interactions}

(A) Quantification of proliferating and senescent myofibroblast volume from immunofluorescent staining against WGA and $\mathrm{YH} 2 \mathrm{AX}$ of frozen tissue sections (left) and adult rabbit cardiac fibroblasts in vitro. (B) Quantification of capacitance of patch clamped proliferating and senescent adult rabbit cardiac myofibroblasts. (C) Modeling of APD90 from a single myocyte coupled to a single proliferating or senescent fibroblast with gradients of myofibroblast conductance (gPro and gSen) and myofibroblast capacitance. (D) Modeling of myocyte (first panel) and myofibroblast (second panel) action potential traces, as well as $I_{\mathrm{Ca}}$ and $\mathrm{I}_{\mathrm{Gap}}$ traces from young and aged modeling conditions. (E) S1S2 modeling of young and aged conditions showing onset of action potential failure with gradients of $\mathrm{Na}$ channel conductance $(\mathrm{gNa})$ and myofibroblast capacitance, with representative action potential traces (left) and onset of action potential failure with gradients of myocyte inward rectifier channel conductance $(\mathrm{gK} 1)$ and myofibroblast capacitance, with representative action potential traces (right)

\section{Supplemental Figure 2: Additional Parameters for Myocyte-Myofibroblast Modeling}


bioRxiv preprint doi: https://doi.org/10.1101/2021.12.21.473692; this version posted December 23, 2021. The copyright holder for this preprint (which was not certified by peer review) is the author/funder, who has granted bioRxiv a license to display the preprint in perpetuity. It is made available under aCC-BY-NC-ND 4.0 International license.

(A) Representative current traces of proliferating and senescent adult rabbit cardiac myofibroblasts at various membrane potentials. (B) I-V curves of cumulative data from proliferating and senescent when the currents shown in $(A)$ were measured at the end of corresponding test pulses. (C) Boltzmann equation parameters used for modeling.

\section{Supplemental Table 1: Antibodies used for Immunofluorescence Staining}

\section{Supplemental Table 2: Rabbit-Specific Primers used for qPCR}

\section{$\underline{\text { References }}$}

1. Benjamin, E.J., et al., Heart Disease and Stroke Statistics-2019 Update: A Report From the American Heart Association. Circulation, 2019. 139(10): p. e56-e528. 
2. Deo, R. and C.M. Albert, Epidemiology and genetics of sudden cardiac death. Circulation, 2012. 125(4): p. 62037.

3. Partridge, L., J. Deelen, and P.E. Slagboom, Facing up to the global challenges of ageing. Nature, 2018. 561(7721): p. 45-56.

4. Kolettis, T.M., Coronary artery disease and ventricular tachyarrhythmia: pathophysiology and treatment. Curr Opin Pharmacol, 2013. 13(2): p. 210-7.

5. Mendonca Costa, C., et al., Modeling the Electrophysiological Properties of the Infarct Border Zone. Front Physiol, 2018. 9: p. 356.

6. van den Borne, S.W., et al., Myocardial remodeling after infarction: the role of myofibroblasts. Nat Rev Cardiol, 2010. 7(1): p. 30-7.

7. Cooper, L.L., et al., Electromechanical and structural alterations in the aging rabbit heart and aorta. Am J Physiol Heart Circ Physiol, 2012. 302(8): p. H1625-35.

8. Cooper, L.L., et al., Redox modification of ryanodine receptors by mitochondria-derived reactive oxygen species contributes to aberrant Ca2+ handling in ageing rabbit hearts. J Physiol, 2013. 591(23): p. 5895-911.

9. Murphy, K.R., et al., Enhancing Autophagy Diminishes Aberrant $\mathrm{Ca}(2+)$ Homeostasis and Arrhythmogenesis in Aging Rabbit Hearts. Front Physiol, 2019. 10: p. 1277.

10. Morrissey, P.J., et al., A novel method of standardized myocardial infarction in aged rabbits. Am J Physiol Heart Circ Physiol, 2017. 312(5): p. H959-H967.

11. Ziv, O., et al., A novel, minimally invasive, segmental myocardial infarction with a clear healed infarct borderzone in rabbits. Am J Physiol Heart Circ Physiol, 2012. 302(11): p. H2321-30.

12. Coppe, J.P., et al., The senescence-associated secretory phenotype: the dark side of tumor suppression. Annu Rev Pathol, 2010. 5: p. 99-118.

13. Rodier, F., et al., Persistent DNA damage signalling triggers senescence-associated inflammatory cytokine secretion. Nat Cell Biol, 2009. 11(8): p. 973-9.

14. Campisi, J. and L. Robert, Cell senescence: role in aging and age-related diseases. Interdiscip Top Gerontol, 2014. 39: p. 45-61.

15. Wang, Y., et al., Restoring expression of wild-type $p 53$ suppresses tumor growth but does not cause tumor regression in mice with a p53 missense mutation. J Clin Invest, 2011. 121(3): p. 893-904.

16. Storer, M., et al., Senescence is a developmental mechanism that contributes to embryonic growth and patterning. Cell, 2013. 155(5): p. 1119-30.

17. Demaria, M., et al., An essential role for senescent cells in optimal wound healing through secretion of PDGF-AA. Dev Cell, 2014. 31(6): p. 722-33.

18. Prata, L., et al., Senescent cell clearance by the immune system: Emerging therapeutic opportunities. Semin Immunol, 2019: p. 101275.

19. Vicente, R., et al., Cellular senescence impact on immune cell fate and function. Aging Cell, 2016. 15(3): p. 400-6.

20. Burton, D.G. and V. Krizhanovsky, Physiological and pathological consequences of cellular senescence. Cell Mol Life Sci, 2014. 71(22): p. 4373-86.

21. Shibamoto, M., et al., Activation of DNA Damage Response and Cellular Senescence in Cardiac Fibroblasts Limit Cardiac Fibrosis After Myocardial Infarction. Int Heart J, 2019. 60(4): p. 944-957.

22. Kirschner, K., et al., Functional heterogeneity in senescence. Biochem Soc Trans, 2020. 48(3): p. 765-773.

23. Biran, A., et al., Senescent cells communicate via intercellular protein transfer. Genes Dev, 2015. 29(8): p. 791802.

24. Baker, D.J., et al., Naturally occurring p16(Ink4a)-positive cells shorten healthy lifespan. Nature, 2016. 530(7589): p. 184-9.

25. Baker, D.J., et al., Clearance of p16Ink4a-positive senescent cells delays ageing-associated disorders. Nature, 2011. 479(7372): p. 232-6.

26. Childs, B.G., et al., Cellular senescence in aging and age-related disease: from mechanisms to therapy. Nat Med, 2015. 21(12): p. 1424-35.

27. Zhu, Y., et al., The Achilles' heel of senescent cells: from transcriptome to senolytic drugs. Aging Cell, 2015. 14(4): p. 644-58. 
28. Chang, J., et al., Clearance of senescent cells by ABT263 rejuvenates aged hematopoietic stem cells in mice. Nat Med, 2016. 22(1): p. 78-83.

29. Kim, E.C. and J.R. Kim, Senotherapeutics: emerging strategy for healthy aging and age-related disease. BMB Rep, 2019. 52(1): p. 47-55.

30. Kirkland, J.L., et al., The Clinical Potential of Senolytic Drugs. J Am Geriatr Soc, 2017. 65(10): p. $2297-2301$.

31. van Deursen, J.M., Senolytic therapies for healthy longevity. Science, 2019. 364(6441): p. 636-637.

32. Kirkland, J.L. and T. Tchkonia, Cellular Senescence: A Translational Perspective. EBioMedicine, 2017. 21: p. $21-28$.

33. Zhu, F., et al., Senescent cardiac fibroblast is critical for cardiac fibrosis after myocardial infarction. PLoS One, 2013. 8(9): p. e74535.

34. Dookun, E., et al., Clearance of senescent cells during cardiac ischemia-reperfusion injury improves recovery. Aging Cell, 2020. 19(10): p. e13249.

35. Francis Stuart, S.D., et al., The crossroads of inflammation, fibrosis, and arrhythmia following myocardial infarction. J Mol Cell Cardiol, 2016. 91: p. 114-22.

36. Aromolaran, A.S., et al., Interleukin-6 inhibition of $h E R G$ underlies risk for acquired long $Q T$ in cardiac and systemic inflammation. PLoS One, 2018. 13(12): p. e0208321.

37. Camelliti, P., et al., Spatially and temporally distinct expression of fibroblast connexins after sheep ventricular infarction. Cardiovasc Res, 2004. 62(2): p. 415-25.

38. Camelliti, P., et al., Fibroblast network in rabbit sinoatrial node: structural and functional identification of homogeneous and heterogeneous cell coupling. Circ Res, 2004. 94(6): p. 828-35.

39. Schultz, F., et al., Cardiomyocyte-myofibroblast contact dynamism is modulated by connexin-43. Faseb j, 2019. 33(9): p. 10453-10468.

40. Axford-Gatley, R.A. and G.J. Wilson, The "border zone" in myocardial infarction. An ultrastructural study in the dog using an electron-dense blood flow marker. Am J Pathol, 1988. 131(3): p. 452-64.

41. Richardson, W.J., et al., Physiological Implications of Myocardial Scar Structure. Compr Physiol, 2015. 5(4): p. 1877-909.

42. Neuschl, V., et al., Structural assessment of myocardial infarction scars and left ventricular function with cardiac magnetic resonance imaging in patients at high risk of sudden cardiac death. Bratisl Lek Listy, 2018. 119(5): p. 259-264.

43. Richardson, W.J. and J.W. Holmes, Why Is Infarct Expansion Such an Elusive Therapeutic Target? J Cardiovasc Transl Res, 2015. 8(7): p. 421-30.

44. Zhang, P., et al., Multiscale Characterization of Impact of Infarct Size on Myocardial Remodeling in an Ovine Infarct Model. Cells Tissues Organs, 2015. 200(5): p. 349-62.

45. Liu, W., et al., Mechanisms linking T-wave alternans to spontaneous initiation of ventricular arrhythmias in rabbit models of long QT syndrome. J Physiol, 2018. 596(8): p. 1341-1355.

46. Fu, X., et al., Specialized fibroblast differentiated states underlie scar formation in the infarcted mouse heart. J Clin Invest, 2018. 128(5): p. 2127-2143.

47. Kanisicak, O., et al., Genetic lineage tracing defines myofibroblast origin and function in the injured heart. Nat Commun, 2016. 7: p. 12260.

48. Prabhu, S.D. and N.G. Frangogiannis, The Biological Basis for Cardiac Repair After Myocardial Infarction: From Inflammation to Fibrosis. Circ Res, 2016. 119(1): p. 91-112.

49. Jun, J.I. and L.F. Lau, Resolution of organ fibrosis. J Clin Invest, 2018. 128(1): p. 97-107.

50. Ito, T., et al., Regulation of Cellular Senescence by Polycomb Chromatin Modifiers through Distinct DNA Damageand Histone Methylation-Dependent Pathways. Cell Rep, 2018. 22(13): p. 3480-3492.

51. Tallquist, M.D., Cardiac Fibroblast Diversity. Annu Rev Physiol, 2020. 82: p. 63-78.

52. Pusztaszeri, M.P., W. Seelentag, and F.T. Bosman, Immunohistochemical expression of endothelial markers CD31, CD34, von Willebrand factor, and Fli-1 in normal human tissues. J Histochem Cytochem, 2006. 54(4): p. 385-95.

53. Pinto, A.R., et al., Revisiting Cardiac Cellular Composition. Circ Res, 2016. 118(3): p. 400-9.

54. Holness, C.L. and D.L. Simmons, Molecular cloning of CD68, a human macrophage marker related to lysosomal glycoproteins. Blood, 1993. 81(6): p. 1607-13.

55. Dai, W., et al., Effect of etoposide-induced alteration of the Mdm2-Rb signaling pathway on cellular senescence in A549 lung adenocarcinoma cells. Oncol Lett, 2017. 14(4): p. 3935-3940. 

available under aCC-BY-NC-ND 4.0 International license.

56. Furman, D., et al., Chronic inflammation in the etiology of disease across the life span. Nat Med, 2019. 25(12): $p$. $1822-1832$.

57. Kabakov, A.Y., et al., Three-week Old Rabbit Ventricular Cardiomyocytes as a Novel System to Study Cardiac Excitation and EC Coupling. Front Physiol, 2021.

58. Narita, M., Juxtacrine regulation of cellular senescence. BMB Rep, 2019. 52(1): p. 3-4.

59. Nelson, G., et al., The senescent bystander effect is caused by ROS-activated NF-kappaB signalling. Mech Ageing Dev, 2018. 170: p. 30-36.

60. Nelson, G., et al., A senescent cell bystander effect: senescence-induced senescence. Aging Cell, 2012. 11(2): $\mathrm{p}$. 345-9.

61. Quinn, T.A., et al., Electrotonic coupling of excitable and nonexcitable cells in the heart revealed by optogenetics. Proc Natl Acad Sci U S A, 2016. 113(51): p. 14852-14857.

62. Kostecki, G.M., et al., Optogenetic current in myofibroblasts acutely alters electrophysiology and conduction of co-cultured cardiomyocytes. Sci Rep, 2021. 11(1): p. 4430.

63. Childs, B.G., T.J. Bussian, and D.J. Baker, Cellular Identification and Quantification of Senescence-Associated betaGalactosidase Activity In Vivo. Methods Mol Biol, 2019. 1896: p. 31-38.

64. Hegyi, B., et al., Complex electrophysiological remodeling in postinfarction ischemic heart failure. Proc Natl Acad Sci U S A, 2018. 115(13): p. E3036-E3044.

65. Walaszczyk, A., et al., Pharmacological clearance of senescent cells improves survival and recovery in aged mice following acute myocardial infarction. Aging Cell, 2019. 18(3): p. e12945.

66. Terentyev, D., et al., Hyperphosphorylation of RyRs underlies triggered activity in transgenic rabbit model of LQT2 syndrome. Circ Res, 2014. 115(11): p. 919-28.

67. Rutherford, S.L., et al., High-resolution 3-dimensional reconstruction of the infarct border zone: impact of structural remodeling on electrical activation. Circ Res, 2012. 111(3): p. 301-11.

68. Di Diego, J.M. and C. Antzelevitch, Ischemic ventricular arrhythmias: experimental models and their clinical relevance. Heart Rhythm, 2011. 8(12): p. 1963-8.

69. Campos, F.O., et al., Factors Promoting Conduction Slowing as Substrates for Block and Reentry in Infarcted Hearts. Biophys J, 2019. 117(12): p. 2361-2374.

70. Anderson, R., et al., Length-independent telomere damage drives post-mitotic cardiomyocyte senescence. Embo j, 2019.

71. Meyer, K., et al., Essential Role for Premature Senescence of Myofibroblasts in Myocardial Fibrosis. J Am Coll Cardiol, 2016. 67(17): p. 2018-28.

72. Kohl, P. and R.G. Gourdie, Fibroblast-myocyte electrotonic coupling: does it occur in native cardiac tissue? J Mol Cell Cardiol, 2014. 70: p. 37-46.

73. Kuilman, T., et al., The essence of senescence. Genes Dev, 2010. 24(22): p. 2463-79.

74. Fischer, A.H., et al., Hematoxylin and eosin staining of tissue and cell sections. CSH Protoc, 2008. 2008: $\mathrm{p}$. pdb.prot4986.

75. Kim, T.Y., et al., Short-Long Heart Rate Variation Increases Dispersion of Action Potential Duration in Long QT Type 2 Transgenic Rabbit Model. Sci Rep, 2019. 9(1): p. 14849.

76. Choi, B.R., W. Jang, and G. Salama, Spatially discordant voltage alternans cause wavebreaks in ventricular fibrillation. Heart Rhythm, 2007. 4(8): p. 1057-68.

77. Kim, T.Y., et al., Complex excitation dynamics underlie polymorphic ventricular tachycardia in a transgenic rabbit model of long QT syndrome type 1. Heart Rhythm, 2015. 12(1): p. 220-8.

78. Brunner, M., et al., Mechanisms of cardiac arrhythmias and sudden death in transgenic rabbits with long $Q T$ syndrome. J Clin Invest, 2008. 118(6): p. 2246-59.

79. Banville, I. and R.A. Gray, Effect of action potential duration and conduction velocity restitution and their spatial dispersion on alternans and the stability of arrhythmias. J Cardiovasc Electrophysiol, 2002. 13(11): p. 1141-9.

80. Hayashi, H., et al., Dynamic origin of spatially discordant alternans in cardiac tissue. Biophys J, 2007. 92 (2): p. 448-60.

81. Dimri, G.P., et al., A biomarker that identifies senescent human cells in culture and in aging skin in vivo. Proc Natl Acad Sci U S A, 1995. 92(20): p. 9363-7. 
bioRxiv preprint doi: https://doi.org/10.1101/2021.12 21.473692; this version posted December 23,2021 . The copyright holder for this preprint (which was not certified by peer review) is the author/funder, who has granted bioRxiv a license to display the preprint in perpetuity. It is made available under aCC-BY-NC-ND 4.0 International license.

82. Debacq-Chainiaux, F., et al., Protocols to detect senescence-associated beta-galactosidase (SA-betagal) activity, a biomarker of senescent cells in culture and in vivo. Nat Protoc, 2009. 4(12): p. 1798-806.

83. Bandyopadhyay, D., et al., Analysis of cellular senescence in culture in vivo: the senescence-associated betagalactosidase assay. Curr Protoc Cell Biol, 2005. Chapter 18: p. 18.9.1-18.9.9.

84. Maity, B., D. Sheff, and R.A. Fisher, Immunostaining: detection of signaling protein location in tissues, cells and subcellular compartments. Methods Cell Biol, 2013. 113: p. 81-105.

85. Pilbrow, A.P., et al., Genomic selection of reference genes for real-time PCR in human myocardium. BMC Med Genomics, 2008. 1: p. 64.

86. Mahajan, A., et al., A rabbit ventricular action potential model replicating cardiac dynamics at rapid heart rates. Biophys J, 2008. 94(2): p. 392-410. 
$*$

A)

\begin{tabular}{c|c|c|c|c|c|c|c|c|}
\multirow{2}{*}{} & Count & \multicolumn{2}{|c|}{ Procedural Deaths } & \multicolumn{2}{c|}{ Procedural Survival } & \multicolumn{2}{c|}{ Sudden Cardiac Death } & Completion \\
\cline { 2 - 10 } Young & & VT/VF & Non-VT/VF & VT/VF & Non-VT/VF & Acute (3-72hrs) & Chronic (>72hrs) & \\
Aged & 93 & 8 & 6 & 9 & 50 & 12 & 2 & 45 \\
\hline Total & 172 & 28 & 8 & 15 & 48 & 15 & 2 & 46 \\
\hline
\end{tabular}

B)

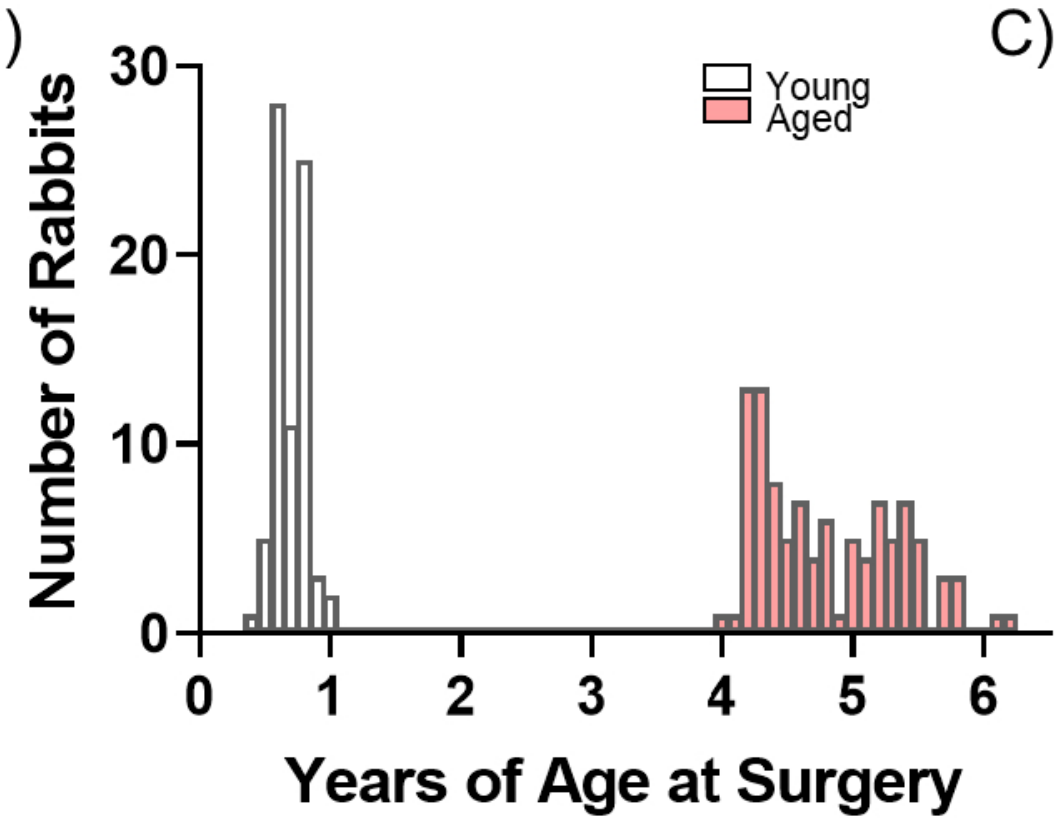

C) VT/VF

$\square$ Non-VT/VF *

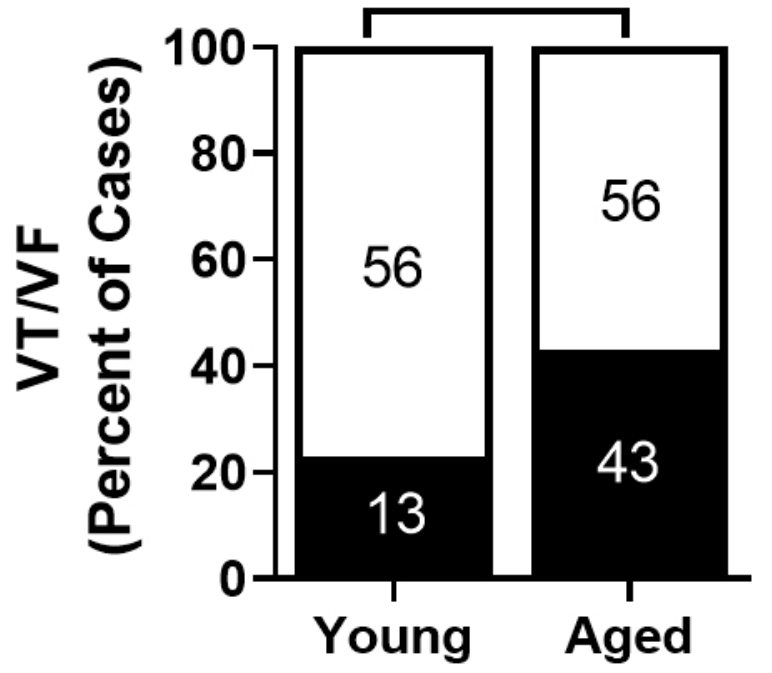

D)

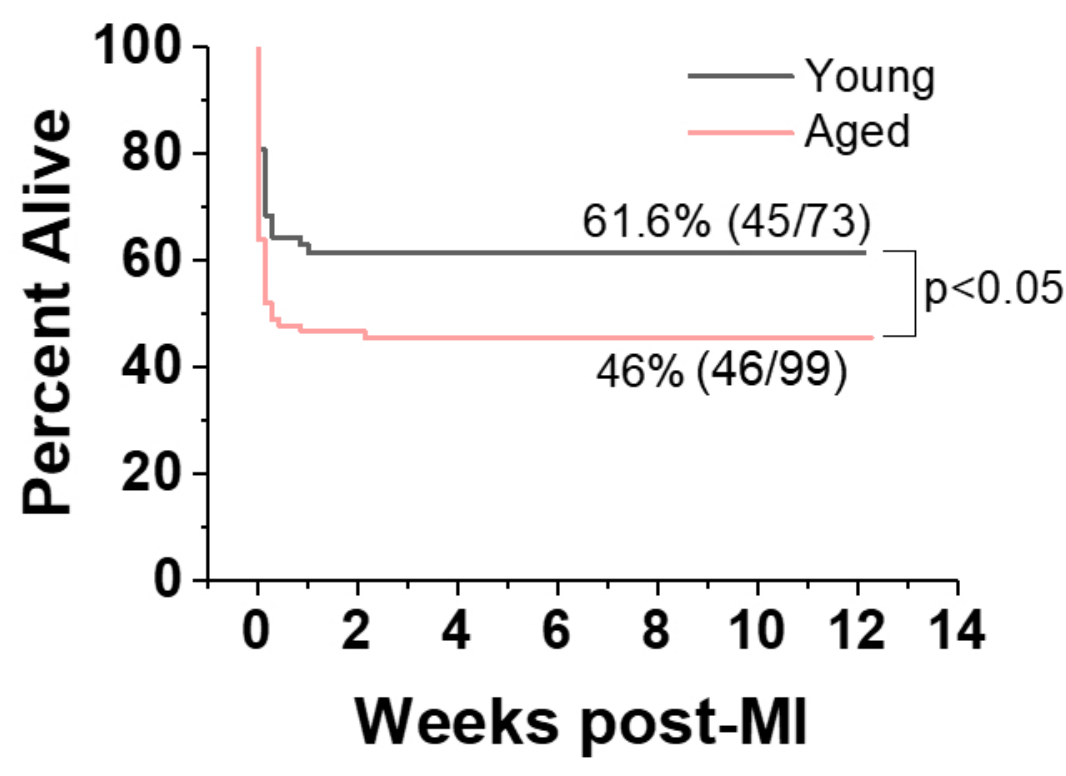



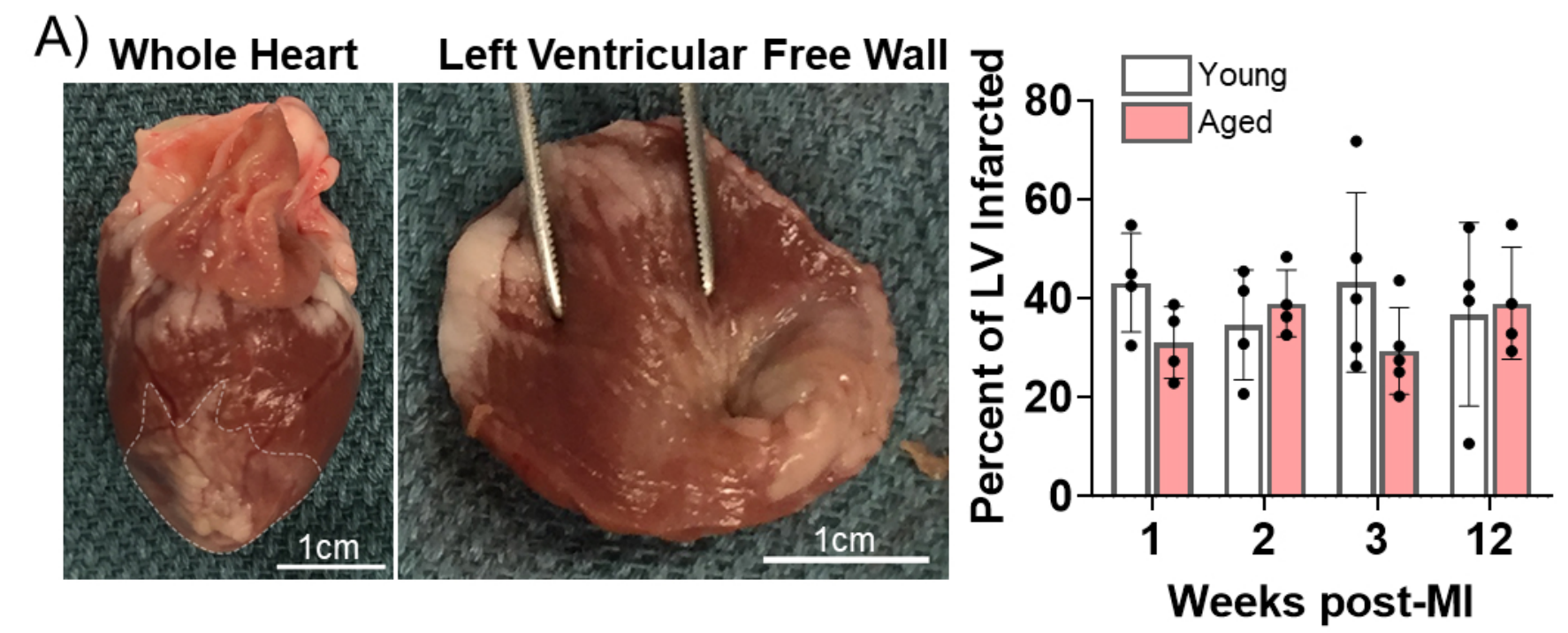

B)

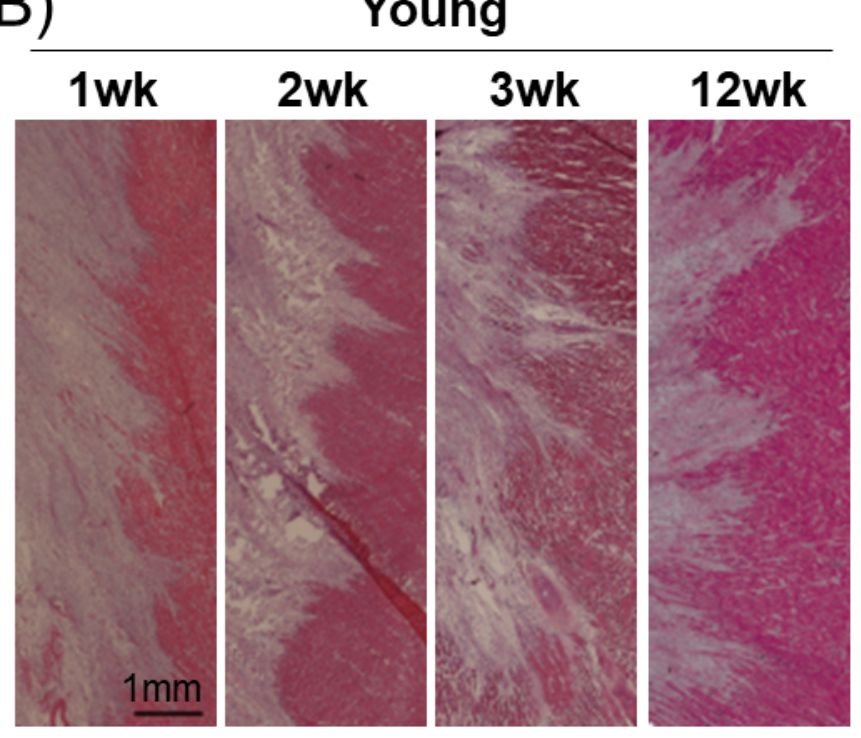

C) Masson's Trichrome

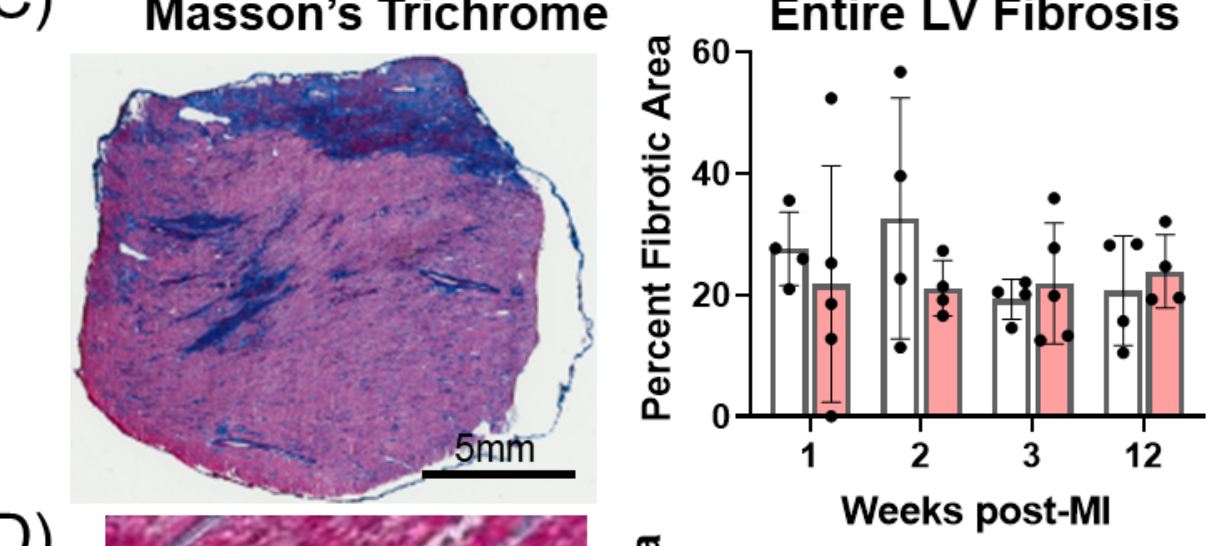

D)
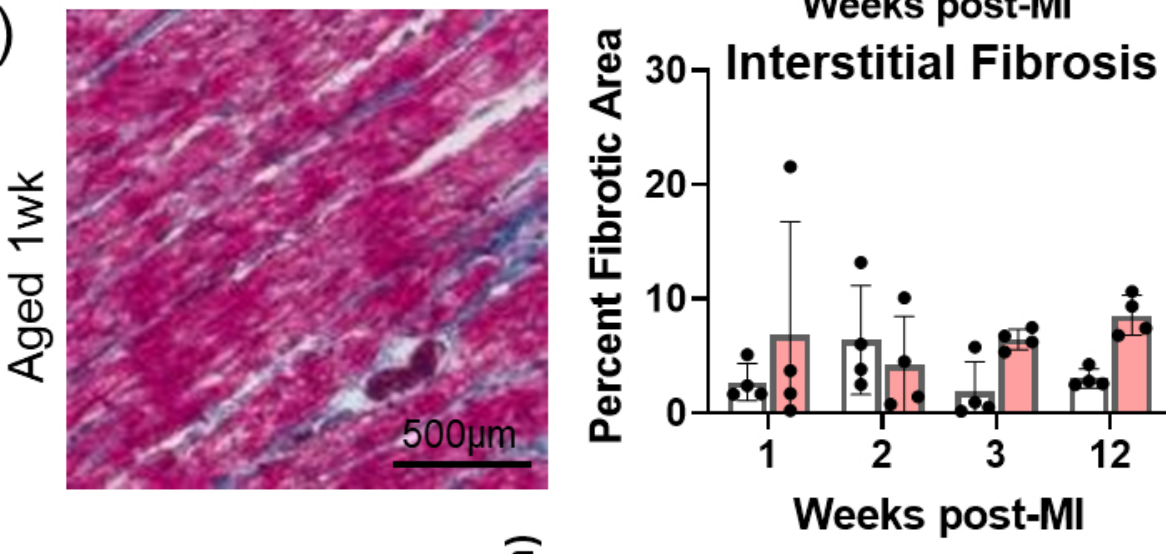
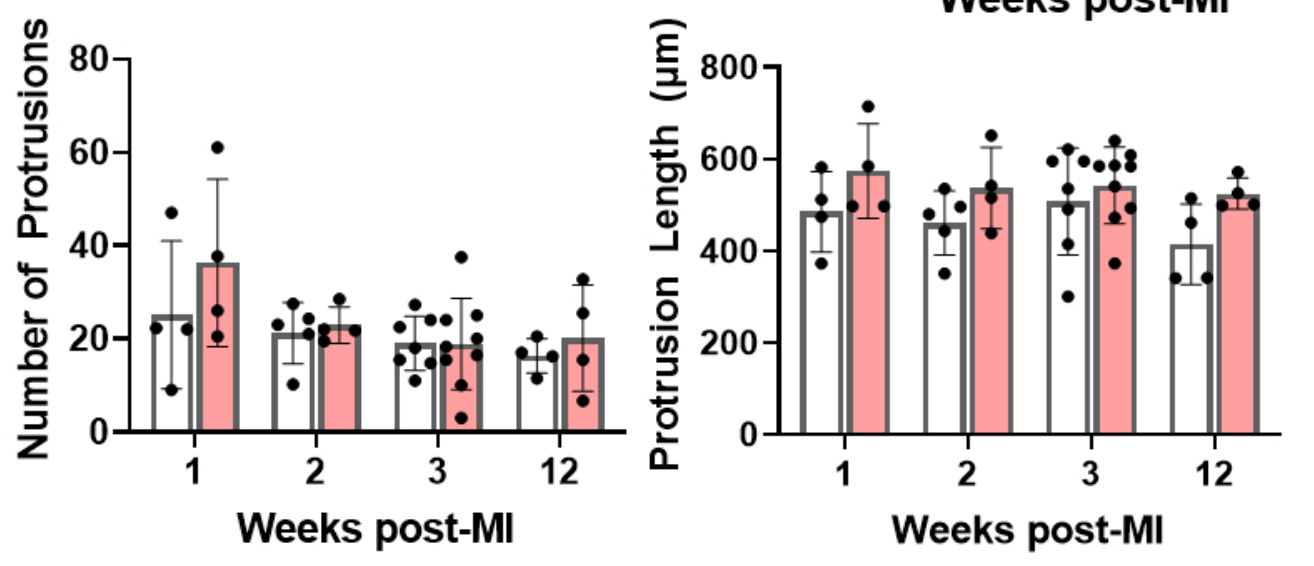


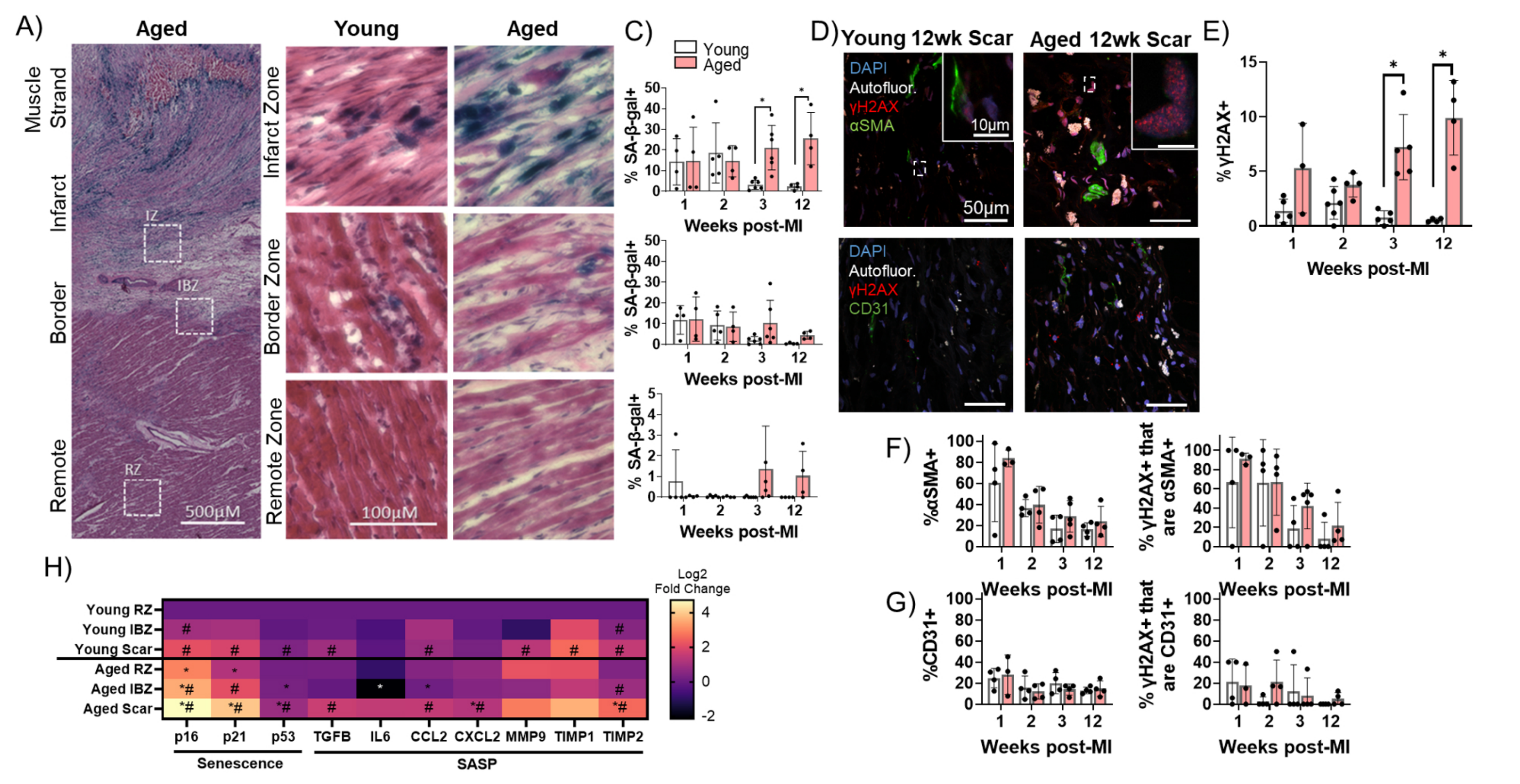



A)

Total current

$5 \mu \mathrm{M}$
$\mathrm{E} 4031$

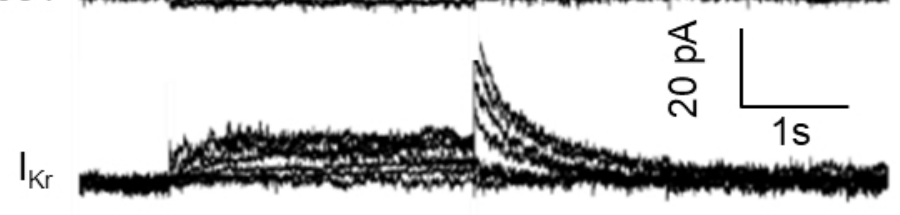

B)

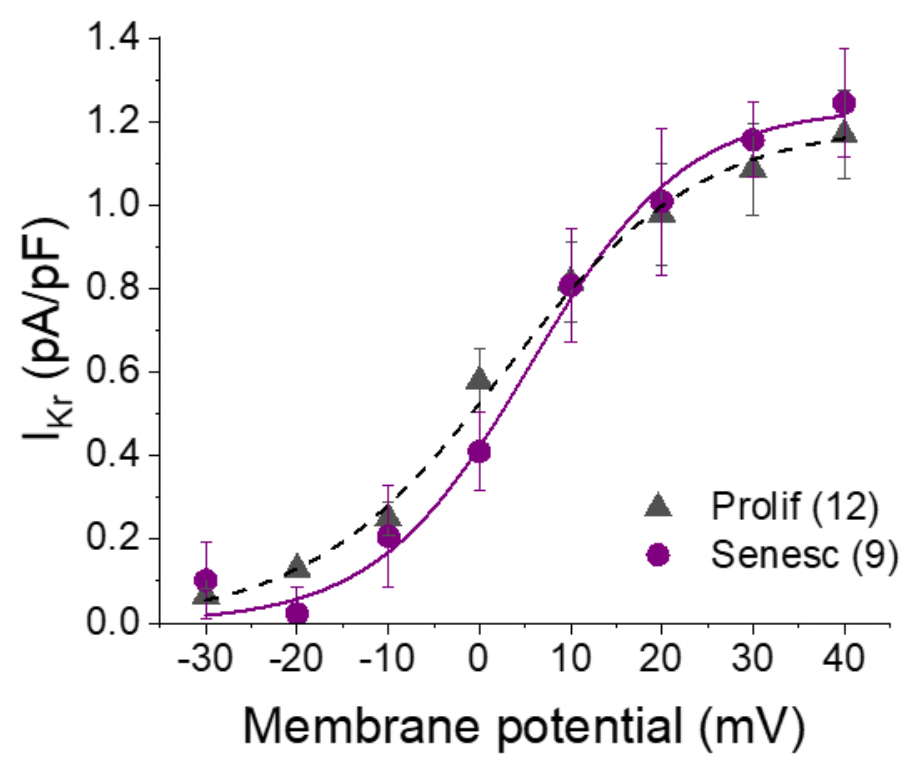

C) Proliferating-CM

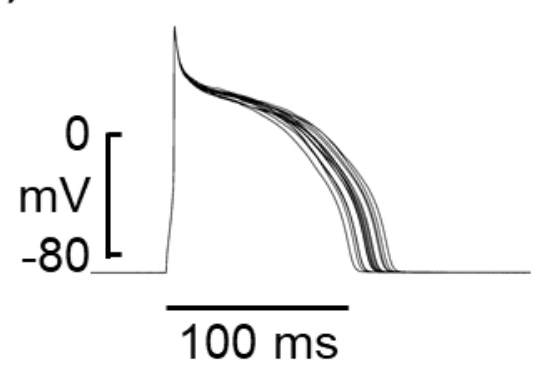

D)

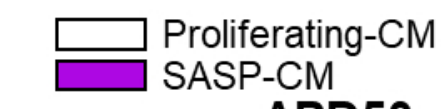

APD50

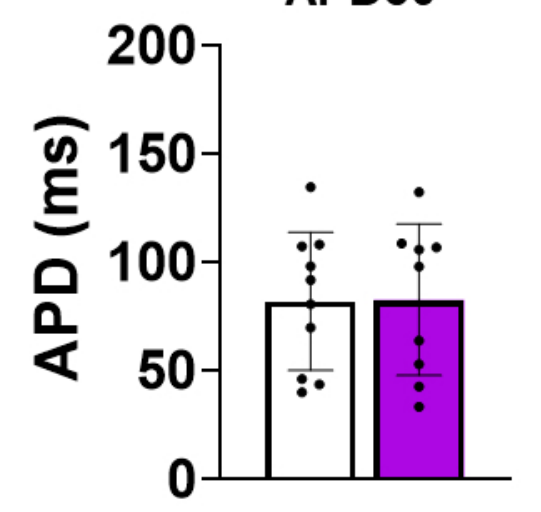

SASP-CM
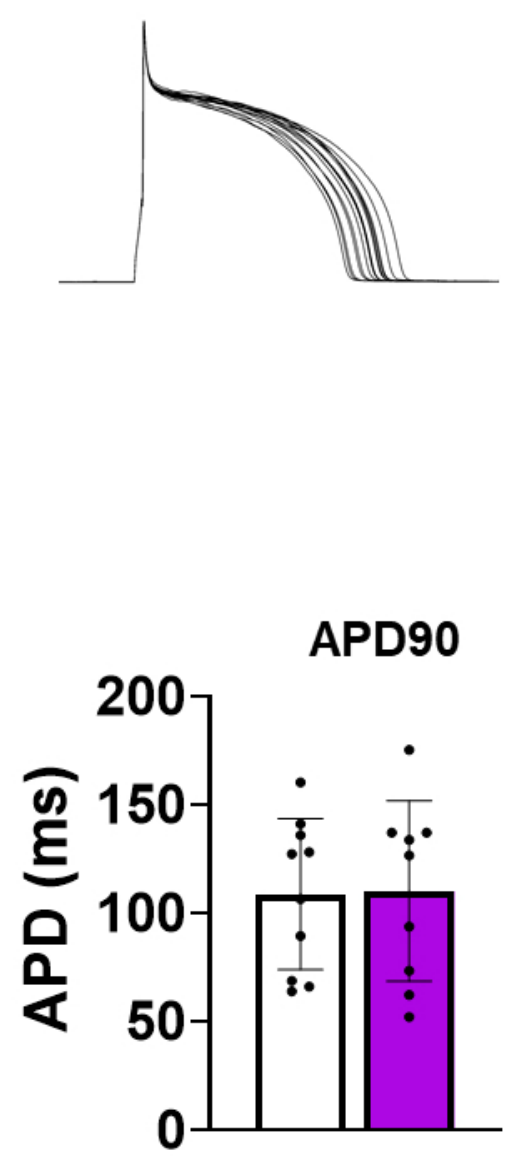


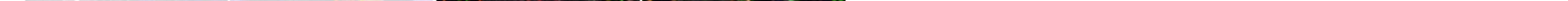


Proliferating

A) Frozen Section

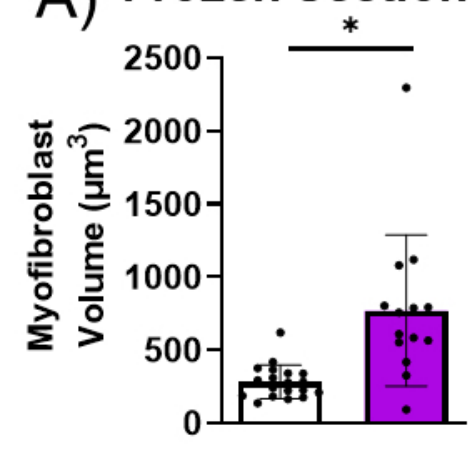
Senescent

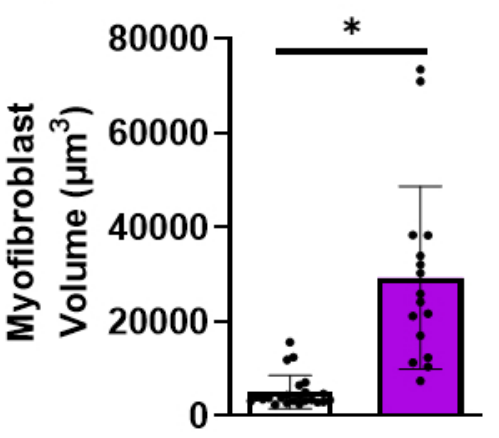

B)

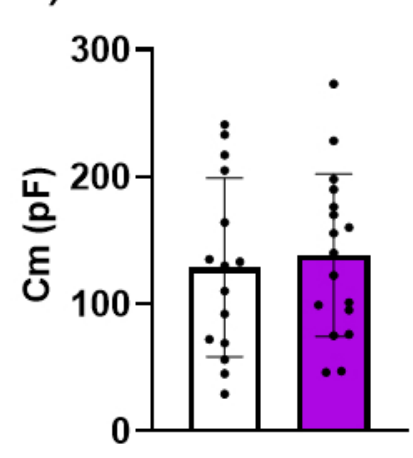

Model: single myofibroblast coupled to myocyte

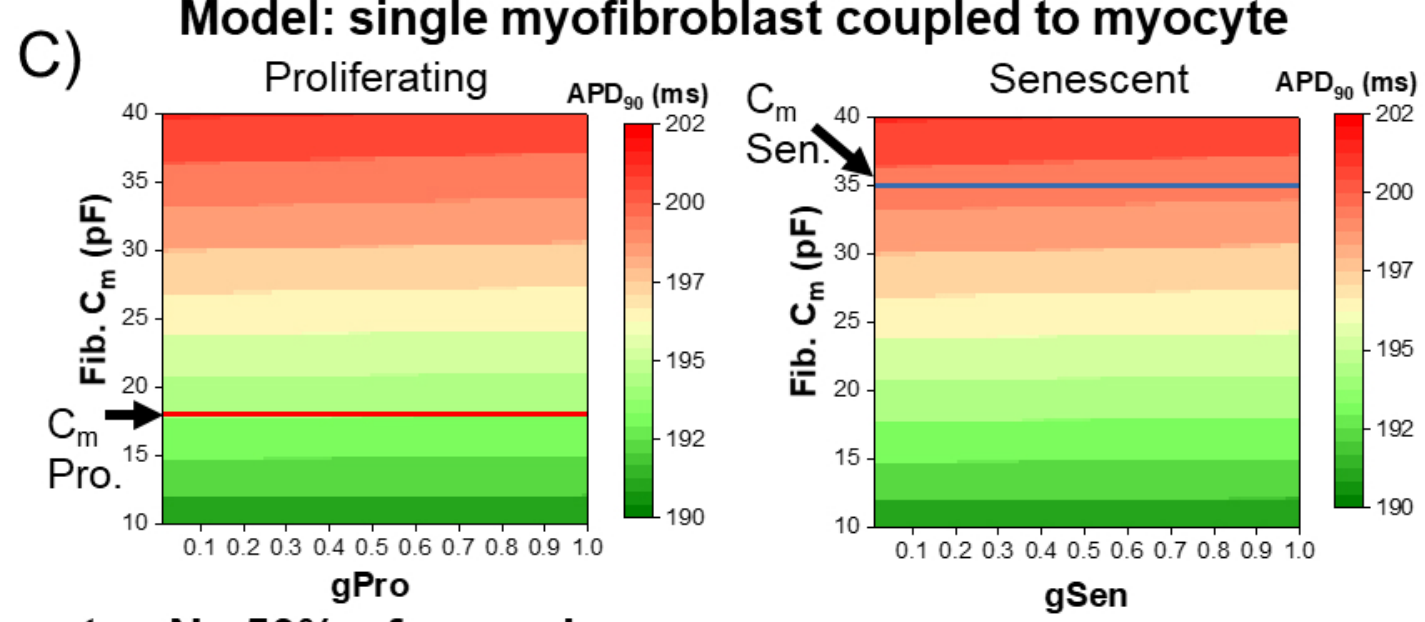

D) Model: multiple myofibroblasts coupled to myocyte, myocyte gNa $50 \%$ of normal

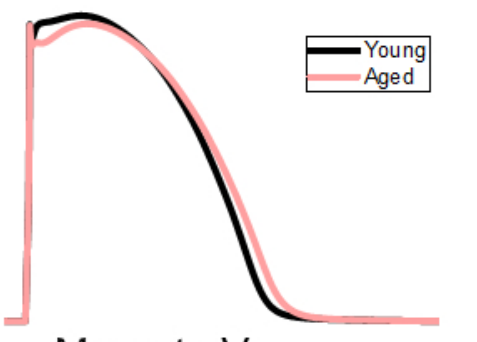

Myocyte $\mathrm{V}_{\mathrm{m}}$
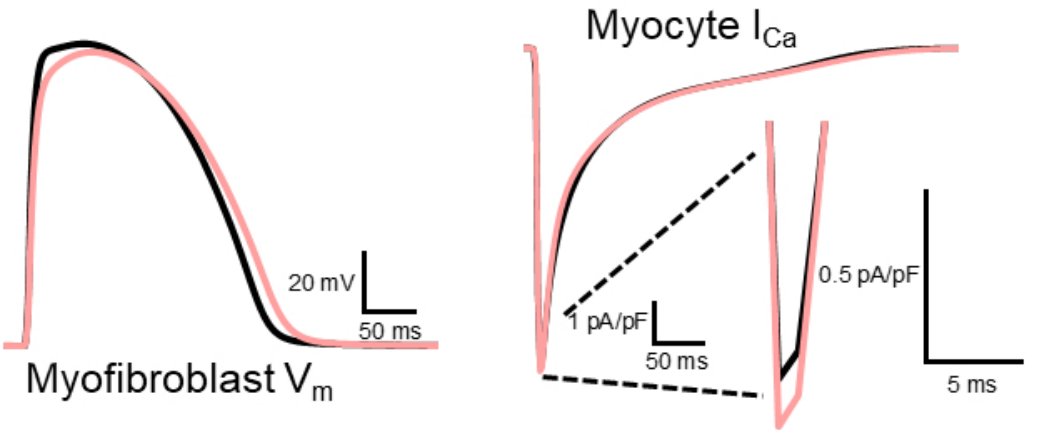

$\mathrm{I}_{\text {Gap }}$

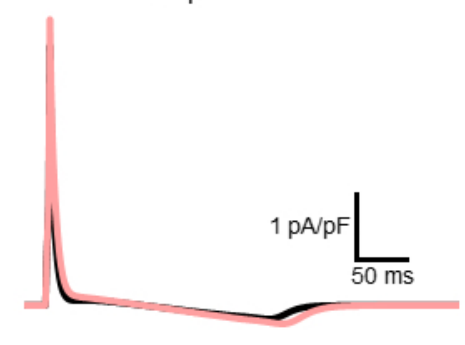

E) Model: multiple myofibroblasts coupled to myocyte, S1S1S1S2 protocol
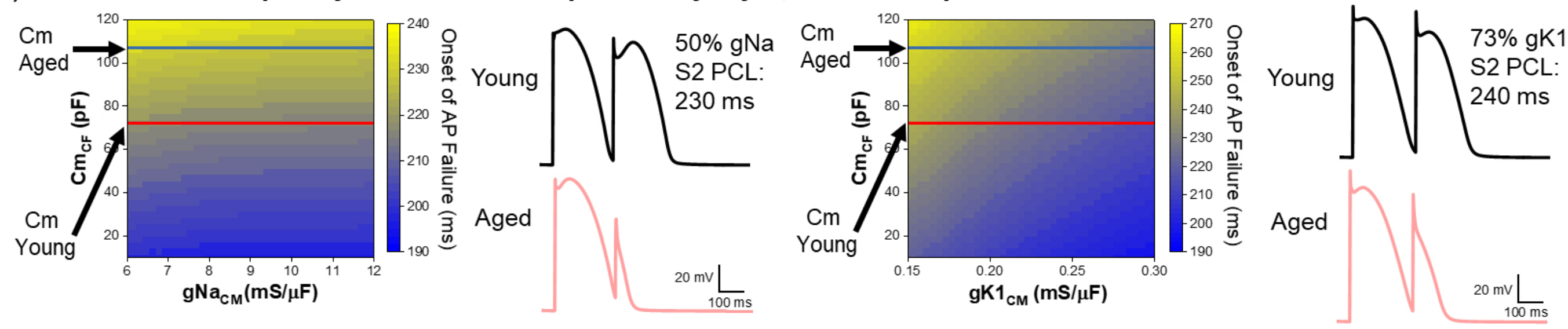